\title{
Accuracy of an efficient framework for structural analysis of wind turbine blades
}

Blasques, José Pedro Albergaria Amaral; Bitsche, Robert D.; Fedorov, Vladimir; Lazarov, Boyan Stefanov

\section{Published in:}

Wind Energy

Link to article, DOI:

10.1002/we.1939

Publication date:

2016

Document Version

Early version, also known as pre-print

Link back to DTU Orbit

\section{Citation (APA):}

Blasques, J. P. A. A., Bitsche, R. D., Fedorov, V., \& Lazarov, B. S. (2016). Accuracy of an efficient framework for structural analysis of wind turbine blades. Wind Energy, 19(9), 1603-1621 . https://doi.org/10.1002/we.1939

\section{General rights}

Copyright and moral rights for the publications made accessible in the public portal are retained by the authors and/or other copyright owners and it is a condition of accessing publications that users recognise and abide by the legal requirements associated with these rights.

- Users may download and print one copy of any publication from the public portal for the purpose of private study or research.

- You may not further distribute the material or use it for any profit-making activity or commercial gain

- You may freely distribute the URL identifying the publication in the public portal 


\title{
Accuracy of an efficient framework for structural analysis of wind turbine blades
}

\author{
J. P. Blasques ${ }^{1}$, R. D. Bitsche ${ }^{1}$, V. Fedorov ${ }^{1}$, B. S. Lazarov ${ }^{2}$ \\ ${ }^{1}$ Department of Wind Energy, Technical University of Denmark, Frederiksborgvej 399, Building 115, 4000 Roskilde, Denmark. \\ ${ }^{2}$ Department of Mechanical Engineering, Technical University of Denmark, Nils Koppels Allé, 2800 Kgs. Lyngby, Denmark.
}

\begin{abstract}
This paper presents a novel framework for the structural design and analysis of wind turbine blades and establishes its accuracy. The framework is based on a beam model composed of two parts - a 2D finite element based cross section analysis tool and a 3D beam finite element model. The cross section analysis tool is able to capture the effects stemming from material anisotropy and inhomogeneity for sections of arbitrary geometry. The proposed framework is very efficient and therefore ideally suited for integration within wind turbine aeroelastic design and analysis tools. A number of benchmark examples are presented comparing the results from the proposed beam model to 3D shell and solid finite element models. The examples considered include a square prismatic beam, an entire wind turbine rotor blade, and a detailed wind turbine blade cross section. Phenomena at both the blade length scale - deformation and eigenfrequencies - and cross section scale - 3D material strain and stress fields - are analyzed. Furthermore, the effect of the different assumptions regarding the boundary conditions is discussed in detail. The benchmark examples show excellent agreement suggesting that the proposed framework is a highly efficient alternative to $3 \mathrm{D}$ finite element models for structural analysis of wind turbine blades.

Copyright (c) 2012 John Wiley \& Sons, Ltd.

\section{KEYWORDS}

wind turbine blade; structural analysis; beam model; cross section analysis

Correspondence

R. D. Bitsche, DTU Wind Energy, Technical University of Denmark, Frederiksborgvej 399, Building 115, 4000 Roskilde, Denmark. E-mail: robi@dtu.dk

Received...
\end{abstract}

\section{INTRODUCTION}

The numerical simulation of the dynamic behavior of wind turbines is typically performed using aeroelastic analysis tools. These tools compute the aerodynamic and structural response of the turbine simultaneously. A large number of dynamic load cases needs to be analyzed for design and certification of wind turbines and the analysis therefore tends to be computationally expensive. As a consequence, wind turbine rotor blades are typically modeled within aeroelastic simulation tools using beam finite elements (see, e.g., [1,2]). This type of elements is specifically developed for the analysis of long and slender structures like wind turbine blades and allows for a good compromise between accuracy and computational efficiency. The accumulated knowledge in aerodynamic and structural design has lead to rotor blades which work closer to their limits while the increasing size of rotor blades has lead to the emergence of new design criteria. As a result, accurate estimates of the turbine response has become paramount leading to a gradual enhancement of the accuracy of beam finite element models [3].

Beam finite element models are generated in two steps. The first step concerns the analysis of the beam cross section properties, e.g., stiffness and mass. In the second step, the beam finite elements are generated through integration of these properties along the beam length. Recent efforts to improve the accuracy of the prediction of the structural response have led to the integration of advanced cross section analysis frameworks within wind turbine aeroelastic simulation tools. The BEam Cross section Analysis Software - BECAS - a framework for structural analysis of wind turbine 
blades, was developed in this context and is presented here. The cross section analysis tool at the core of BECAS is an implementation of the theory originally presented by Giavotto et al. [4] for the analysis of inhomogeneous anisotropic beams. Implementations of this theory have been used as a benchmark for the validation of new cross section analysis tools emerging since the early 1980's, namely, VABS which is perhaps currently the most widely used cross section analysis tool (see, e.g., [5, 6, 7]). Among other, BECAS can take into account the effects of material anisotropy (from, e.g., fiber orientation) and correctly estimate torsional stiffness, a feature previously deemed unimportant when analyzing shorter blades. These new features make it possible to incorporate new blade technologies like aeroelastic tailoring for load mitigation through material and geometrical design. The internal forces and moments at each section of the blade stemming from nonlinear aeroelastic analysis can be seamlessly transferred to the cross section analysis tool. It is then possible to accurately assess the structural performance of the blade with a high level of detail down to the material level with a very low computational effort.

Applications of BECAS include the multimaterial structural topology optimization framework presented in [8,9] for optimal design of laminated composite beam cross sections. A novel approach for the computation of strain energy release rates in fractured beams using the Virtual Crack Closure Technique within BECAS was recently presented in [10]. BECAS was also used extensively for the design of the DTU 10MW reference wind turbine [11, 12]. The current paper builds on the experience gained throughout this project, which led to a number of improvements of the tool and a number of developments related to automatic pre- and post-processing. The workflow devised in the project and employed in the current paper is described in Figure 1. The pre-processing step concerns the generation of input for the cross section analysis tool based on existing information of the blade. BECAS currently encompasses a number of solutions for automatic input generation based on, e.g., existing shell finite element models. The stiffness and mass properties are then computed by the cross section analysis tool and used as input for the wind turbine aeroelastic simulation tool (e.g., HAWC2 [2]). The internal cross section forces and moments resulting from wind turbine aeroelastic simulations are finally returned to BECAS, and used, e.g., for the analysis of the local strain and stress fields at a cross section level.

The accuracy of a number of beam modeling methods has been previously assessed in terms of the beam displacements and rotations in $[13,14]$ for beams with simple geometries. The cross section stiffness and mass properties obtained by a number of different tools, some of which specifically developed for wind turbine blade analysis, have been compared in [7]. Initial results concerning the validation of the local stresses and strains based on the theories underlying VABS and BECAS have been presented in [15] and [4], respectively. The comprehensive validation work presented in this paper extends the existing body of work in this field by presenting a set of results in which BECAS is compared against 3D shell and solid finite element models of realistic wind turbine blade designs. The validation considers cross section stiffness and mass properties, static deformation and eigenfrequencies, and local strains and stresses at the material level. The results presented will allow blade designers to understand the level of accuracy and the benefits and limitations of the proposed framework for the specific problem at hand.

The paper is divided as follows. The theoretical methodology underlying the BECAS framework is described in the next section. Afterwards, the setup for each of the numerical experiments used for validation is described and the results are presented. The results are interpreted and discussed in the next section. Finally, the merits of the proposed framework are discussed in the last section.

\section{METHODOLOGY}

The methodology employed for the analysis of the structural response of the beams is described in this section. A general beam finite element model is described first. The BECAS framework for analysis of anisotropic beam cross sections with arbitrary geometry is described next. Finally, details regarding the solution procedure and implementation are discussed.

\subsection{Beam finite element analysis}

The beam finite element static equilibrium equations are given as (cf. [16])

$$
\hat{\mathbf{K}} \hat{\mathbf{u}}=\hat{\mathbf{f}} \quad \text { where } \quad \hat{\mathbf{K}}=\sum_{e=1}^{n_{b}} \hat{\mathbf{K}}_{e}=\sum_{e=1}^{n_{b}} \int_{0}^{L_{e}} \hat{\mathbf{B}}_{e}^{T} \mathbf{K}_{s} \hat{\mathbf{B}}_{e} \mathrm{~d} z
$$

where $n_{b}$ is the number of elements in the beam finite element assembly, $L_{e}$ is the length of element $e$, and the

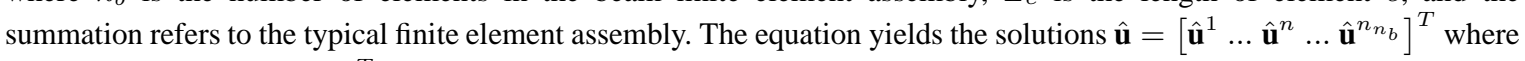
$\hat{\mathbf{u}}^{n}=\left[\begin{array}{llllll}\hat{u}_{x}^{n} & \hat{u}_{y}^{n} & \hat{u}_{z}^{n} & \hat{r}_{x}^{n} & \hat{r}_{y}^{n} & \hat{r}_{z}^{n}\end{array}\right]^{T}$ are the three displacements $u$ and rotations $r$ with respect to the $x, y$, and $z$ axis of node

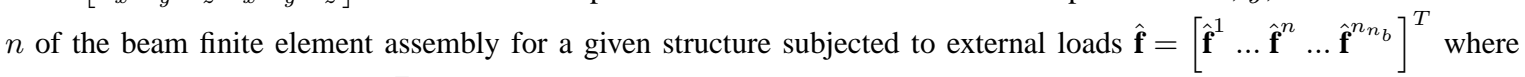
$\hat{\mathbf{f}}^{n}=\left[\begin{array}{llllll}\hat{f}_{x}^{n} & \hat{f}_{y}^{n} & \hat{f}_{z}^{n} \hat{m}_{x}^{n} \hat{m}_{y}^{n} \hat{m}_{z}^{n}\end{array}\right]^{T}$ are the three forces $\hat{f}$ and moments $\hat{m}$ with respect to the $x, y$, and $z$ axis of node $n$, 
where $n_{n_{b}}$ is the number of nodes in the beam finite element assembly. The beam finite element stiffness matrix $\hat{\mathbf{K}}_{e}$ is given in function of the cross section stiffness matrix $\mathbf{K}_{s}$ and the strain-displacement matrix $\hat{\mathbf{B}}_{e}=\hat{\mathcal{B}}(\hat{\mathbf{N}})$ ), where $\hat{\mathcal{B}}$ is the strain-displacement relation. The shape function matrix $\hat{\mathbf{N}}_{e}$ is defined such that $\mathbf{r}=\hat{\mathbf{N}} \hat{\mathbf{u}}$ where $\mathbf{r}=\left[\chi^{T} \varphi^{T}\right]^{T}$ is composed of the three translations in $\chi=\left[\begin{array}{lll}\chi_{x} & \chi_{y} & \chi_{z}\end{array}\right]^{T}$ of the cross section reference point, and three rotations in $\varphi=\left[\begin{array}{lll}\varphi_{x} & \varphi_{y} & \varphi_{z}\end{array}\right]^{T}$. Herein, four node beam finite elements with cubic Lagrangean interpolation functions are used.

\subsection{Cross section analysis}

The accuracy of the beam finite element model depends mainly on the accuracy of the cross section stiffness properties. For a linear elastic beam there exists a linear relation between the internal cross section forces $\mathbf{T}=\left[T_{x} T_{y} T_{z}\right]^{T}$ and moments $\mathbf{M}=\left[\begin{array}{lll}M_{x} & M_{y} M_{z}\end{array}\right]^{T}$ in $\boldsymbol{\theta}=\left[\mathbf{T}^{T} \mathbf{M}^{T}\right]^{T}$ (cf. Figure 2(a)), and internal strains $\boldsymbol{\tau}=\left[\tau_{x} \tau_{y} \tau_{z}\right]^{T}$ and curvatures $\boldsymbol{\kappa}=\left[\begin{array}{lll}\kappa_{x} & \kappa_{y} & \kappa_{z}\end{array}\right]^{T}$ in $\boldsymbol{\psi}=\left[\boldsymbol{\tau}^{T} \boldsymbol{\kappa}^{T}\right]^{T}$ (cf. Figure 2(b)). This relation is given in its stiffness form as

$$
\boldsymbol{\theta}=\mathbf{K}_{s} \boldsymbol{\psi}
$$

where $\mathbf{K}_{s}$ is the $6 \times 6$ cross section stiffness matrix. In the most general case, considering material anisotropy and inhomogeneity, all 21 stiffness parameters in $\mathbf{K}_{s}$ may be required to describe the deformation of the beam cross section. The methodology described next allows the accurate determination of all entries of $\mathbf{K}_{s}$. The beams may have an arbitrary cross section geometry while the materials may be anisotropic and inhomogeneously distributed in the cross section.

\subsubsection{Beam kinematics and finite element formulation}

The strains and stresses acting at a point in the cross section of the beam are $\epsilon=\left[\epsilon_{x x} \epsilon_{y y} 2 \epsilon_{x y} 2 \epsilon_{x z} 2 \epsilon_{y z} \epsilon_{z z}\right]^{T}$,

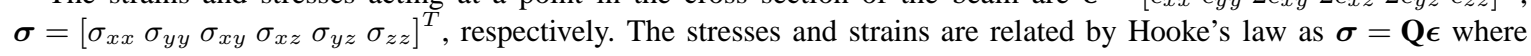
$\mathbf{Q}$ is the $6 \times 6$ material constitutive matrix. The forces and moments in $\boldsymbol{\theta}$ are statically equivalent to the stress components $\mathbf{p}=\left[\sigma_{x z} \sigma_{y z} \sigma_{z z}\right]^{T}$ acting on the cross section such that $\boldsymbol{\theta}=\int_{A} \mathbf{Z}^{T} \mathbf{p} \mathrm{dA}$ where $\mathrm{A}$ is the cross section area, and

$$
\mathbf{Z}=\left[\mathbf{I}_{3} \mathbf{n}\right], \text { with } \mathbf{n}=\left[\begin{array}{ccc}
0 & -z & y \\
z & 0 & -x \\
-y & x & 0
\end{array}\right]
$$

and $\mathbf{I}_{3}$ is an identity matrix of size $3 \times 3$. The coordinates $x$ and $y$ define the location of a point in the cross section given with respect to the cross section reference coordinate system (cf. Figure 2).

It is assumed herein that the total displacement $\mathbf{s}=\left[\begin{array}{lll}s_{x} & s_{y} & s_{z}\end{array}\right]^{T}$ of a point in the cross section is obtained as $\mathbf{s}=\mathbf{v}+\mathbf{g}$ (see Figure 3). The displacements $\mathbf{v}=\left[\begin{array}{lll}v_{x} & v_{y} & v_{z}\end{array}\right]^{T}$ are associated with the rigid body translations and rotations of the cross section in $\mathbf{r}$ through $\mathbf{v}=\mathbf{Z r}$. The displacements $\mathbf{g}=\left[\begin{array}{lll}g_{x} & g_{y} & g_{z}\end{array}\right]^{T}$ are associated with the in- and out-of-plane cross section distortion henceforth referred to as warping displacements. These are approximated following the typical finite element approach such that $\mathbf{g} \approx \mathbf{N u}$ where $\mathbf{N}$ is the matrix of finite element shape functions and $\mathbf{u}$ the nodal warping displacements. In practice the cross section geometry is discretized using two dimensional finite elements with three degrees of freedom per node associated with the three dimensional nodal warping displacements $u_{x}, u_{y}$, and $u_{z}$. From the definitions above the total displacement of a point in the cross section is finally defined as

$$
\mathbf{s}=\mathbf{Z r}+\mathbf{N u}
$$

A formula for the strains at a point in the cross section can now be derived based on the displacement definition presented above. Assuming small strains, $\epsilon_{\alpha \beta}=1 / 2\left(\partial s_{\alpha} / \partial \beta+\partial s_{\beta} / \partial \alpha\right),(\alpha, \beta=x, y, z)$ is employed thus yielding

$$
\boldsymbol{\epsilon}=\mathbf{S Z} \psi+\mathbf{B u}+\mathbf{S N} \frac{\partial \mathbf{u}}{\partial z}
$$

where $\boldsymbol{\psi}=\left(\mathbf{T}_{r}+\frac{\partial}{\partial z}\right) \mathbf{r}$ where $\mathbf{T}_{r} \mathbf{r}=\left[\begin{array}{lllll}0 & 0 & 0 & \tau_{y}-\tau_{x} & 0\end{array}\right]$. These in fact correspond to the typical beam theory result in which $\tau_{x}=\partial \chi_{x} / \partial z-\varphi_{y}, \tau_{y}=\partial \chi_{y} / \partial z+\varphi_{x}, \tau_{z}=\partial \chi_{x} / \partial z, \kappa_{x}=\partial \varphi_{x} / \partial z, \kappa_{y}=\partial \varphi_{y} / \partial z$, and $\kappa_{z}=\partial \varphi_{z} / \partial z$. Finally, the strain-displacement matrices are defined as $\mathbf{B}=\mathcal{B}(\mathbf{N})$ where

$$
\mathcal{B}=\left[\begin{array}{cccccc}
\partial / \partial x & 0 & \partial / \partial y & 0 & 0 & 0 \\
0 & \partial / \partial y & \partial / \partial x & 0 & 0 & 0 \\
0 & 0 & 0 & \partial / \partial x & \partial / \partial y & 0
\end{array}\right]^{T}
$$

and $\mathbf{S}=\left[\begin{array}{ll}\mathbf{0} & \mathbf{I}_{3}\end{array}\right]$ in which $\mathbf{0}_{3}$ and $\mathbf{I}_{3}$ are the $3 \times 3$ zero and identity matrices, respectively. This corresponds to separating the derivatives $\partial / \partial z$ and leaving them unsolved. Note that henceforth ()$_{\partial z}=\frac{\partial()}{\partial z}$ for clarity. 


\subsubsection{Virtual work principle}

The first variation of the total virtual work per unit length $\delta W$ of two sections of the beam with a vanishing distance between them is

$$
\delta W=\delta W_{e}+\delta W_{i}=0
$$

where $W_{i}$ is the work done by the internal elastic forces, and $W_{e}$ the work done by the external forces acting on the cross section. The variation of the internal strain energy is given by

$$
\delta W_{i}=\int_{\Omega} \delta \boldsymbol{\epsilon}^{T} \boldsymbol{\sigma} d \Omega=\left[\begin{array}{c}
\delta \mathbf{u}_{\partial z} \\
\delta \mathbf{u} \\
\delta \boldsymbol{\psi}
\end{array}\right]^{T}\left[\begin{array}{ccc}
\mathbf{M} & \mathbf{C} & \mathbf{L} \\
\mathbf{C}^{T} & \mathbf{E} & \mathbf{R} \\
\mathbf{L}^{T} & \mathbf{R}^{T} & \mathbf{A}
\end{array}\right]\left[\begin{array}{c}
\mathbf{u}_{\partial z} \\
\mathbf{u} \\
\boldsymbol{\psi}
\end{array}\right]
$$

where the strain-displacement relation derived in (5) was invoked. Each of the system matrices presented above is defined as

$$
\begin{aligned}
\underset{(6 \times 6)}{\mathbf{A}} & =\sum_{e=1}^{n_{e}} \int_{A} \mathbf{Z}_{e}^{T} \mathbf{S}_{e}^{T} \mathbf{Q}_{e} \mathbf{S}_{e} \mathbf{Z}_{e} \mathrm{dA}, \underset{\left(n_{d} \times 6\right)}{\mathbf{R}}=\sum_{e=1}^{n_{e}} \int_{A} \mathbf{B}_{e}^{T} \mathbf{Q}_{e} \mathbf{S}_{e} \mathbf{Z}_{e} \mathrm{dA}, \underset{\left(n_{d} \times n_{d}\right)}{\mathbf{E}}=\sum_{e=1}^{n_{e}} \int_{A} \mathbf{B}_{e}^{T} \mathbf{Q}_{e} \mathbf{B}_{e} \mathrm{dA}, \\
\underset{\left(n_{d} \times n_{d}\right)}{\mathbf{C}} & =\sum_{e=1}^{n_{e}} \int_{A} \mathbf{B}_{e}^{T} \mathbf{Q}_{e} \mathbf{S}_{e} \mathbf{N}_{e} \mathrm{dA}, \underset{\left(6 \times n_{d}\right)}{\mathbf{L}}=\sum_{e=1}^{n_{e}} \int_{A} \mathbf{Z}_{e}^{T} \mathbf{S}_{e}^{T} \mathbf{Q}_{e} \mathbf{S}_{e} \mathbf{N}_{e} \mathrm{dA}, \underset{\left(n_{d} \times n_{d}\right)}{\mathbf{M}}=\sum_{e=1}^{n_{e}} \int_{A} \mathbf{N}_{e}^{T} \mathbf{S}_{e}^{T} \mathbf{Q}_{e} \mathbf{S}_{e} \mathbf{N}_{e} \mathrm{dA}
\end{aligned}
$$

where $e$ is the element number and $n_{e}$ is the number of finite elements in the cross section mesh. The total number of degrees of freedom associated with the cross section finite element mesh is $n_{d}=n_{n} \times 3$ where the number of nodes $n_{n}$ multiplies the number of degrees of freedom at each node. The sums in (9) refer to the typical assembly procedure used in finite element analysis.

Assuming that surface and body forces are not present, the only contribution to the variation of the external work $\delta W_{e}$ stems from the tractions which act on the cross section face. After some manipulation (see [17]) it can be shown that

$$
\delta W_{e}=\int_{A} \frac{\partial\left(\delta \mathbf{s}^{T} \mathbf{p}\right)}{\partial z} \mathrm{dA}=\left[\begin{array}{c}
\delta \mathbf{u}_{\partial z} \\
\delta \mathbf{u} \\
\delta \boldsymbol{\psi}
\end{array}\right]^{T}\left[\begin{array}{c}
\mathbf{P} \\
\mathbf{P}_{\partial z} \\
\boldsymbol{\theta}
\end{array}\right]+\delta \mathbf{r}^{T}\left(\boldsymbol{\theta}_{\partial z}-\mathbf{T}_{r}^{T} \boldsymbol{\theta}\right)
$$

where $\mathbf{P}=\int \mathbf{N}^{T} \mathbf{p} \mathrm{dA}, \mathbf{P}_{\partial z}=\int \mathbf{N}^{T} \mathbf{p}_{\partial z}$ dA. The vector $\mathbf{P}$ can be seen as the nodal stresses in the cross section finite element discretization as it represents the discretized stresses acting on the cross section face.

Equations (7) and (10) must be valid for any $\delta \mathbf{u}_{\partial z}, \delta \mathbf{u}$, and $\delta \boldsymbol{\psi}$, and thus from (6) the variation of the total potential energy is finally restated as

$$
\left[\begin{array}{ccc}
\mathbf{M} & \mathbf{C} & \mathbf{L} \\
\mathbf{C}^{T} & \mathbf{E} & \mathbf{R} \\
\mathbf{L}^{T} & \mathbf{R}^{T} & \mathbf{A}
\end{array}\right]\left[\begin{array}{c}
\mathbf{u}_{\partial z} \\
\mathbf{u} \\
\boldsymbol{\psi}
\end{array}\right]=\left[\begin{array}{c}
\mathbf{P} \\
\mathbf{P}_{\partial z} \\
\boldsymbol{\theta}
\end{array}\right] \text { and } \boldsymbol{\theta}_{\partial z}=\mathbf{T}_{r}^{T} \boldsymbol{\theta}
$$

Differentiating the first row with respect to $z$ and adding it to the second yields the following set of second order linear partial differential equations

$$
\left\{\begin{array}{r}
\mathbf{M} \mathbf{u}_{\partial^{2} z}+\left(\mathbf{C}-\mathbf{C}^{T}\right) \mathbf{u}_{\partial z}+\mathbf{L} \boldsymbol{\psi}_{\partial z}-\mathbf{E} \mathbf{u}-\mathbf{R} \boldsymbol{\psi}=\mathbf{0} \\
\mathbf{L}^{T} \mathbf{u}_{\partial z}+\mathbf{R}^{T} \mathbf{u}+\mathbf{A} \boldsymbol{\psi}=\boldsymbol{\theta} \\
\boldsymbol{\theta}_{\partial z}=\mathbf{T}_{r}^{T} \boldsymbol{\theta}
\end{array}\right.
$$

This second order linear differential equation renders two types of solutions - an homogeneous and particular solutions - corresponding to two different physical phenomena. The homogeneous solution $(\boldsymbol{\theta}=0)$ is associated with the deformations at the ends or extremities of the beam. The particular solution $(\boldsymbol{\theta} \neq 0)$, on the other hand, will yield the displacement field at the central part of the beam where end effects become negligible. We focus on the latter as this will serve as the basis for the generation of beam finite elements suitable for structural analysis of relatively long and slender beams.

\subsubsection{Cross section equilibrium equations}

First note that the definition of the displacements in (4) is six times redundat. The six rigid body motions - three translations and three rotations - already represented by the shear and curvatures in $\mathbf{r}$ can also be represented by the warping displacements $\mathbf{u}$. A series of constraints are therefore introduced through the Lagrange multiplier method. The 
variation of the virtual work expression in (6) is consequently augmented to include

$$
\delta W_{c}=\int \delta \boldsymbol{\lambda}_{\mathbf{u}}^{T} \mathbf{Z u d A}+\int \boldsymbol{\lambda}_{\mathbf{u}}^{T} \mathbf{Z} \delta \mathbf{u} \mathrm{dA}+\int \delta \boldsymbol{\lambda}_{\mathbf{u}_{\partial z}}^{T} \mathbf{Z} \mathbf{u}_{\partial z} \mathrm{dA}+\int \boldsymbol{\lambda}_{\mathbf{u}_{\partial z}}^{T} \mathbf{Z} \delta \mathbf{u}_{\partial z} \mathrm{dA}
$$

where $\boldsymbol{\lambda}_{\mathbf{u}}$ and $\boldsymbol{\lambda}_{\mathbf{u}_{\partial z}}$ are the Lagrange multipliers associated with $\mathbf{u}$ and $\mathbf{u}_{\partial z}$, respectively. This can be written in matrix form as $\mathbf{D}=\left[\begin{array}{lll}\mathbf{Z}_{1} \ldots \mathbf{Z}_{n_{n}}\end{array}\right]$ where $\mathbf{Z}_{n}$ is obtained from (3) evaluated at the nodal coordinates of node $n$.

Taking into account the constraint above and after manipulation of (12) the resulting equilibrium equations for a section in the central part of the beam are defined in matrix form as

$$
\mathbf{K w}=\mathbf{f} \Leftrightarrow\left[\begin{array}{cc}
\mathbf{K}_{11} & \mathbf{K}_{12} \\
\mathbf{0} & \mathbf{K}_{11}
\end{array}\right]\left[\begin{array}{l}
\mathbf{w}_{1} \\
\mathbf{w}_{2}
\end{array}\right]=\left[\begin{array}{l}
\mathbf{f}_{1} \\
\mathbf{f}_{2}
\end{array}\right] \text { where } \mathbf{K}_{11}=\left[\begin{array}{ccc}
\mathbf{E} & \mathbf{R} & \mathbf{D} \\
\mathbf{R}^{T} & \mathbf{A} & \mathbf{0} \\
\mathbf{D}^{T} & \mathbf{0} & \mathbf{0}
\end{array}\right], \mathbf{K}_{12}=\left[\begin{array}{ccc}
\left(\mathbf{C}^{T}-\mathbf{C}\right) & -\mathbf{L} & \mathbf{0} \\
\mathbf{L}^{T} & \mathbf{0} & \mathbf{0} \\
\mathbf{0} & \mathbf{0} & \mathbf{0}
\end{array}\right]
$$

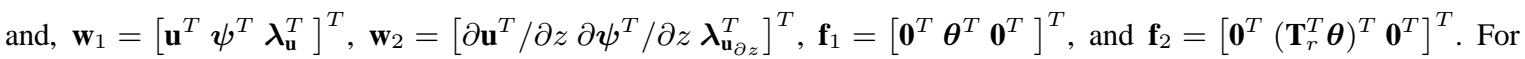
given internal section forces and moments $\boldsymbol{\theta}$ the set of equations above yields the warping displacements $\mathbf{u}$ and internal strains $\psi$, respectively.

The cross section compliance matrix $\mathbf{F}_{s}$ can be determined based on the cross section equilibrium equations presented above. The first step consists of solving the set of equations in (14) for six different right-hand sides each corresponding to setting one of the entries of $\boldsymbol{\theta}$ to unity and the remaining to zero. This corresponds to solving the following set of equations

$$
\mathbf{K W}=\mathbf{F} \Leftrightarrow\left[\begin{array}{cc}
\mathbf{K}_{11} & \mathbf{K}_{12} \\
\mathbf{0} & \mathbf{K}_{11}
\end{array}\right]\left[\begin{array}{l}
\mathbf{W}_{1} \\
\mathbf{W}_{2}
\end{array}\right]=\left[\begin{array}{l}
\mathbf{F}_{1} \\
\mathbf{F}_{2}
\end{array}\right]
$$

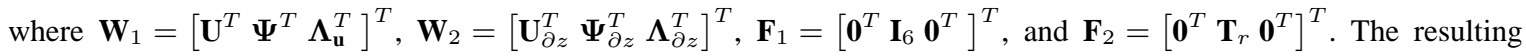
solution matrices $\mathbf{U}, \mathbf{U}_{\partial z}, \Psi$ and $\Psi_{\partial z}$ have six columns corresponding to each of the right-hand sides. For any given $\boldsymbol{\theta}$ the solutions $\mathbf{w}_{1}$ and $\mathbf{w}_{2}$ can be obtained from the linear combination of the columns of $\mathbf{W}_{1}$ and $\mathbf{W}_{2}$ as $\mathbf{w}_{1}=\mathbf{W}_{1} \boldsymbol{\theta}$ and $\mathbf{w}_{2}=\mathbf{W}_{2} \boldsymbol{\theta}$, respectively.

Equating the balance between the complimentary form of the cross section external energy and the internal elastic strain energy yields

$$
\delta \boldsymbol{\theta}^{T} \mathbf{F}_{s} \boldsymbol{\theta}=\delta \boldsymbol{\theta}^{T} \mathbf{W}^{T} \mathbf{G W} \boldsymbol{\theta}
$$

where

$$
\mathbf{G}=\left[\begin{array}{ll}
\mathbf{G}_{11} & \mathbf{G}_{12} \\
\mathbf{G}_{12}^{T} & \mathbf{G}_{22}
\end{array}\right] \text {, and } \mathbf{G}_{11}=\left[\begin{array}{ccc}
\mathbf{E} & \mathbf{R} & \mathbf{0} \\
\mathbf{R}^{T} & \mathbf{A} & \mathbf{0} \\
\mathbf{0} & \mathbf{0} & \mathbf{0}
\end{array}\right], \mathbf{G}_{12}=\left[\begin{array}{ccc}
\mathbf{C} & \mathbf{L} & \mathbf{0} \\
\mathbf{L}^{T} & \mathbf{0} & \mathbf{0} \\
\mathbf{0} & \mathbf{0} & \mathbf{0}
\end{array}\right], \text { and } \mathbf{G}_{22}=\left[\begin{array}{ccc}
\mathbf{M} & \mathbf{0} & \mathbf{0} \\
\mathbf{0} & \mathbf{0} & \mathbf{0} \\
\mathbf{0} & \mathbf{0} & \mathbf{0}
\end{array}\right]
$$

The expression for the cross section compliance matrix is readily obtained from (16) as

$$
\mathbf{F}_{s}=\mathbf{W}^{T} \mathbf{G W}
$$

It is safe to assume that for most relevant structural analysis problems $\mathbf{F}_{s}$ is positive definite and thus the cross section stiffness matrix $\mathbf{K}_{s}$ is simply given by $\mathbf{K}_{s}=\mathbf{F}_{s}^{-1}$.

\subsubsection{Strain and stress evaluation}

Assuming that the internal cross section forces and moments $\boldsymbol{\theta}$ have been previously determined, e.g., from the beam finite element solution or from simple static equilibrium considerations. Assuming also that the warping solutions to the unit loads $\mathbf{U}, \mathbf{U}_{\partial z}$, and $\boldsymbol{\Psi}$ in (15) have been previously determined and are readily available. The strains $\boldsymbol{\epsilon}_{e}$ at element $e$ in the cross section coordinate system are then calculated as

$$
\boldsymbol{\epsilon}_{e}=\mathbf{S}_{e} \mathbf{Z}_{e} \boldsymbol{\Psi}_{e} \boldsymbol{\theta}+\mathbf{B}_{e} \mathbf{U}_{e} \boldsymbol{\theta}+\mathbf{S}_{e} \mathbf{N}_{e} \mathbf{U}_{\partial z, e} \boldsymbol{\theta}
$$

where the subscript $e$ indicates the element number. The strains may be evaluated at different positions in each element. In the current implementation of BECAS, the strains and stresses can be evaluated at element centers or Gauss point position, i.e., $\mathbf{S}_{e}, \mathbf{Z}_{e}, \mathbf{B}_{e}$ and $\mathbf{N}_{e}$ are evaluated at these locations. The arrays $\mathbf{U}_{e}, \mathbf{U}_{\partial z, e}$, and $\boldsymbol{\Psi}_{e}$ are obtained by extracting the degrees of freedom of element $e$ from the corresponding arrays. Finally, the stresses are obtained through $\boldsymbol{\sigma}_{e}=\mathbf{Q}_{e} \boldsymbol{\epsilon}_{e}$ where $\mathbf{Q}_{e}$ is the $6 \times 6$ material constitutive matrix. 
The six components of stress and strains are herein defined with respect to three different coordinate systems, namely, element, fiber plane, and fiber coordinate systems as described in Figure 4. The element coordinate system $\left(x_{e}, y_{e}, z_{e}\right)$ is parallel to the cross section coordinate system. The fiber plane coordinate system $\left(x_{p}, y_{p}, z_{p}\right)$ is defined such that the $x_{p}-z_{p}$ plane is parallel to the stacking plane of the laminate. It is obtained through a rotation $\alpha_{p}$ of the element coordinate system where $\alpha_{p}$ is denominated the fiber plane angle. Finally, the fiber coordinate system $\left(x_{f}, y_{f}, z_{f}\right)$ is associated with the principal material directions so it corresponds to the typical material coordinate system. It results from a rotation $\alpha_{f}$ of the fiber plane coordinate system where $\alpha_{f}$ is referred to as the fiber angle. In sum, any material orientation in 3D can be defined by the angles $\alpha_{p}$ and $\alpha_{f}$. These coordinate systems and angles are referred to later in Section 3 and 4 when presenting and discussing the validation results.

\subsection{Solution procedure and implementation}

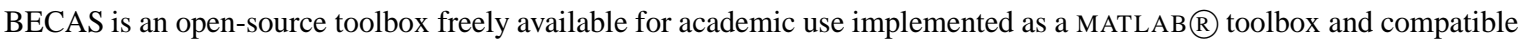
with OCTAVE. All details of the implementation are discussed in [17]. In practice the procedure for the evaluation of $\mathbf{K}_{s}$ consists of first assembling the matrices in (9) using standard finite element techniques. The cross section finite element discretization is based on two dimensional isoparametric finite elements with three degrees of freedom at each node. The next step consists of assembling the matrices $\mathbf{K}_{11}$ and $\mathbf{K}_{12}$ in (14), and finding the solutions to (15). BECAS uses sparse storage for reducing memory requirements. The linear system of equations in (15) is solved by LU factorization of the coefficient matrices. The Schur complement method is employed where a block of matrix $\mathbf{K}_{11}$ is defined such that the factorization is performed on a matrix with reduced bandwidth. The Cuthill-McKee reordering scheme is implemented in order to further reduce bandwidth. Finally, having assembled matrix $\mathbf{G}$, the cross section compliance matrix $\mathbf{F}_{s}$ is computed by replacing the solutions obtained into (18) and $\mathbf{K}_{s}$ is finally obtained.

As an example, consider a problem with a cross section discretization corresponding to 200000 degrees of freedom. In its current implementation, BECAS v2.3, takes approximately 40 seconds to determine the cross section stiffness and mass properties (including assembly and solution times) on a Dell Latitude with 4GB of RAM and two Intel i5-2520M CPUs at 2.50GHz. Models with 1.3 million degrees of freedom have been solved successfully in about 7 minutes on the same platform thus attesting the efficient use of memory.

\section{NUMERICAL EXAMPLES}

The results obtained using the beam finite element framework based on the proposed cross section analysis tool are presented and validated in this section. The general setup for the validation work is presented first and each of the numerical examples is described in detail next.

\subsection{Setup}

Three different numerical examples were considered as presented in Table I. In the first validation example a solid prismatic beam with square cross section made from an orthotropic material was considered. Three different orientations of the orthotropic material were analyzed. This is an academic example which serves to illustrate general features of the proposed model. Among other, it is demonstrated that the effects of material anisotropy and resulting couplings are correctly captured both at the beam length scale (tip displacement and eigenfrequencies) and cross section scale (local stresses). Furthermore, the effect of the boundary conditions were studied in order to establish the consequences of the assumptions underlying the beam finite element model.

The second example considers the DTU 10MW reference wind turbine rotor blade presented in [11, 12]. BECAS was extensively used in the design of this blade. Hence, the results presented here serve to further establish the accuracy of the proposed method when used within such wind turbine blade design frameworks. Again, results are presented associated both with the blade length scale (blade displacements and eigenfrequencies) and cross section scale (local strains along the caps and trailing edge panels).

Finally, in the last example, the interest was on the detailed analysis of local phenomena at the cross section scale, namely, strains and stresses at the material level. In this example the geometry, materials distribution, and structural layout of a wind turbine cross section were defined in great detail. This example serves to establish the accuracy of the proposed method when working at later stages of the design process where a great level of detail has been reached.

For all numerical experiments the displacements and rotations were assumed small and varying linearly with respect to the loads. The structural stiffness, frequency, and strength responses were analyzed using beam finite element models generated based on BECAS, henceforth referred to as BECAS models. The results were compared against detailed 3D finite element models discretized using shell and solid finite elements. The material properties and load cases for each of the validation examples are compiled in Tables II and III, respectively. 


\subsection{Prismatic beam of square cross-section}

A $2 \mathrm{~m}$ long cantilevered beam with square cross section of $0.1 \mathrm{~m}$ by $0.1 \mathrm{~m}$ was considered. The cross section coordinate system is presented in Figure 5 where $z$ is the longitudinal axis. The beam is composed of unidirectional carbon-fiber reinforced plastics (UD CFRP) whose mechanical properties are listed in Table II. Three different material orientations were considered, described in terms of the fiber angle $\alpha_{a}$ and fiber plane angle $\alpha_{p}$ defined in Figure 4. In the first case, S1, all fibers were aligned with the beam axis and stacked in an horizontal plane $\left(\alpha_{a}=0^{\circ}, \alpha_{p}=0^{\circ}\right)$ parallel to the $x-z$ plane. In case S2 all fibers were oriented at $17.5^{\circ}$ while the stacking plane remained horizontal $\left(\alpha_{a}=17.5^{\circ}, \alpha_{p}=0^{\circ}\right)$. Finally, in case S3 the fibers were oriented at $17.5^{\circ}$ with respect to the beam longitudinal axis and stacked in a plane rotated by $17.5^{\circ}$ around the $z$ axis $\left(\alpha_{a}=17.5^{\circ}, \alpha_{p}=17.5^{\circ}\right)$.

The structural response (tip displacement, natural frequencies, and stresses) of the beam was analyzed using a beam finite element model. The stiffness and mass properties were determined using BECAS where the cross section was discretized using $20 \times 20$ eight-node quadratic plane finite elements with three degrees of freedom per node (see Figure 5). The resulting stiffness and mass matrices were integrated along the length using 20 straight, four-node cubic beam finite elements. The nodal displacement and rotations of the beam finite element model were constrained at the clamped end of the beam.

The results were compared against a 3D solid finite element model (3DFEM) generated in ABAQUS using 20-node solid finite elements (C3D20) [18]. Here the beam was discretized using 20x20 elements in the cross section plane and 101 elements along the length of the beam corresponding to a total of 525615 degrees of freedom. Boundary conditions and loads were applied to the 3D solid finite element model using two master nodes. One master node was placed at the center of the section at the clamped end of the beam, while the other master node was placed at the center of the section at the opposite end of the beam where the loads were applied. The master nodes were coupled to the nodes representing the respective cross section via so-called coupling constraints. Two different types of coupling constraint were used kinematic and distributing. Kinematic coupling constraints force the cross section nodes to move like a rigid body and therefore prevent warping deformation of the cross section. Distributing coupling constraints constrain the motion of the cross section nodes to the rigid body motion defined by the master node in an average sense and therefore do not prevent warping deformation of the cross section [18]. In order to study the effect of the different boundary conditions, two model variations were considered. The first model used a kinematic coupling constraint at the clamped end and a distributing coupling constraint at the opposite end where the loads were applied. This model is henceforth referred to as fixed-free. The second model used a distributing coupling constraint at both ends of the beam and is henceforth referred to as free-free.

Beam tip displacements and rotations, along with the five lowest natural frequencies are compared in Table IV for each of the model configurations. The magnitude of the resulting stresses obtained using BECAS and 3DFEM are compared in Figure 6. Results are evaluated at a path along the length of the beam and in more detail at a section in the middle of the beam (1 meter away from both ends). The position of the path is indicated in Figure 5. The path results obtained using BECAS are compared to the free-free and fixed-free results from 3DFEM. At the central section of the beam the BECAS results are compared with free-free 3DFEM results.

\subsection{DTU 10MW Reference Wind Turbine Rotor Blade}

The $86.37 \mathrm{~m}$ long rotor blade of the DTU 10 MW reference wind turbine (DTU10MW RWT) was considered here. The reader is referred to $[11,12]$ for an extensive description of the structural design of the blade including details on geometry, material properties and structural topology, among other. The slight pre-bend of the blade described in [11, 12] was not considered in either of the models described here.

A total of 51 cross sections along the blade length were defined as presented in Figure 7 . The cross section coordinate system of each section was placed at the half-chord point with the axis parallel to the blade coordinate system shown in Figure 7. For the development of the BECAS based beam finite element model the stiffness and mass properties were analyzed at each of these sections. The cross section finite element mesh was generated automatically based on the shell finite element model as shown in Figure 9. The beam finite element model of the blade was then obtained by integration of these properties. A total of 52 straight beam finite elements located along the $Z$ axis of the blade coordinate system (see Figure 7) were used.

The results obtained using the BECAS model were compared against the ABAQUS 3D shell finite element model (3DFEM). The shell finite elements were placed in the outer surface of the aerodynamic shell. The nodes were offset to the outer surface everywhere except the shear webs, where they were placed at the mid-thickness position. The layup was defined at each element using the layering capabilities of the S8R shell finite element in ABAQUS. Two sets of master nodes were defined at the half-chord position at each of the 51 cross sections. The first set was coupled to the cross section nodes representing the load carrying caps using a distributing coupling constraint (see Section 3.2 for a description of the different types of constraints). These nodes were used for application of the loads. The second set of master nodes was instead connected to all nodes in the section. These master nodes were used for measuring the displacements and rotations of each cross section which were then compared to the beam finite element results. The decision for using two different 
sets of nodes for load and deformation resulted from the fact that the rotation results, namely the torsional rotation $r_{z}$, showed much larger deviations when determined only based on the nodes in the caps. The reason is that relative local deformation of the caps due to the loading has a strong influence on the estimated torsional rotation.

Two load cases were considered - BLC1 and BLC2 as described in Table III. In load case BLC1 the blade was loaded by 11 concentrated forces -6 forces in the flapwise direction $\left(f_{y}\right)$ and 5 forces in the edgewise direction $\left(f_{x}\right)$. The forces were defined in such a way that the resulting distribution of bending moments closely approximates the distribution of the ultimate bending moments resulting from the aeroelastic computations as described in $[11,12]$. The forces were applied at the half-chord point at each section. In the BECAS based beam finite element model the loads were applied as point forces and moments at the beam nodes which are located along the $Z$ axis of the blade coordinate system. A torsional moment $m_{z}$ was therefore included together with the forces in order to account for the offset between the half-chord point and the position of the beam node at each cross section (see Table III).

In load case BLC2 a torsional moment was applied at the tip of the blade. The ability to correctly estimate the torsion response was one of the main motivations for the development of BECAS. Hence this load case was introduced so that the accuracy of the torsional response of the wind turbine blade could be assessed individually.

The most relevant components of displacements and rotations at each of the 51 sections calculated using both BECAS and the 3D shell finite element model are compared in Figure 8 for both load cases. The strains were measured along three different paths defined along the length of the blade as indicated in Figure 10. The relevant components of the strain are compared in Figure 11 for both load cases. Finally, the six lowest natural frequencies are compared in Table V.

\subsection{Detailed wind turbine blade cross-section}

In this last validation example we focused on the analysis of the strains and stresses on a generic wind turbine blade cross section where the structural lay-out was defined with great detail. The cross section geometry, finite element mesh, coordinate system, and location of reference, shear, mass, and elastic centers along with the elastic axes as determined by BECAS are presented in Figure 12. The mechanical properties of the materials UD, BIAX, TRIAX, Core, and Adhesive are given in Table II. The outer shell consisted of a sandwich structure with an inner core material (Core) and faces composed of a tri-axial laminate (TRIAX). The suction and pressure side shells were bonded to each other and to the load carrying spar by an adhesive (Adhesive). The spar caps consisted mainly of uni-directional fibers (UD) while the shear webs were sandwich structures with a core material (Core) and layers of a bi-axial laminate (BIAX) in the faces. The material distribution at a detail of the junction between shear web and the cap is presented in Figure 15(a). The cross section geometry and structural lay-out were defined with great detail and a complete description of the model within this paper is therefore impractical. Instead the authors ensure the reproducibility of the results by making the input files to this numerical example available upon request.

Two load cases were considered in this validation example - DLC1 and DLC2 as defined in Table III. In load case DLC1 the cross section was subjected to a transverse force $T_{y}=1 \times 10^{6} \mathrm{~N}$ and a bending moment $M_{x}=-30 \times 10^{6} \mathrm{~N} \mathrm{~m}$. This is similar to subjecting the blade to a flapwise load. In load case DLC2 the cross section was subjected to a torsional moment $M_{z}=1 \times 10^{6} \mathrm{Nm}$. As previously mentioned, torsion load cases were introduced to ascertain the accuracy of BECAS when predicting the response of beams in torsion, an important motivation for the development of these type of numerical tools.

In BECAS the cross section was meshed using 8 node quadratic plane elements with three degrees of freedom per node (see Figure 12). As no results associated with the beam length scale (beam displacements or eigenfrequencies) were computed, a beam finite element model was unnecessary.

The results from BECAS were compared against a 3D solid finite element model in ABAQUS. The finite element mesh was generated through extrusion of the cross section mesh presented in Figure 12. The result was a $60 \mathrm{~m}$ long solid finite element model of a beam of constant cross section meshed with 20 node layered solid finite elements (ABAQUS element type C3D20). The model was clamped at one end while tip forces and moments were applied at the opposite end. The loading was chosen such that it induces the same internal forces and moments as in DLC1 and DLC2 at the cross section of interest, i.e., at the central section of the beam, $30 \mathrm{~m}$ from the ends, where the strains and stresses were analyzed. The length was chosen to ensure that the effect of the boundary conditions does not affect the results.

The strains and stresses estimated by both numerical models were finally compared. The six stress components were evaluated along paths defined in Figure 13. The results obtained from each model for different paths, load cases, and stress components are presented in Figure 14. Finally, the six components of the strains at a detail of the connection between the shear web and the cap were analyzed. The shear strain $\epsilon_{12}$ in the material coordinate system resulting from the DLC1 load case is compared in Figure 15(b). 


\section{DISCUSSION}

In general the tip deformation and eigenfrequencies for the square prismatic beam S1, S2, and S3 in Table IV show very good agreement with the 3DFEM results. The effect of the unconstrained warping boundary conditions assumed in the beam finite element model is clearly visible in the results. The beam finite element model matches very closely the 3DFEM model with free-free boundary conditions, the largest deviation being $0.14 \%$ and $0.99 \%$ for the tip deformation and eigenfrequencies, respectively. The deviation is larger when comparing to the 3DFEM model with fixed-free boundary conditions, where the tip deformation results suggest that beam finite element model tends to underestimate the bending stiffness. That is, the constrained warping boundary condition has a stiffening effect on the beam. The largest deviation in this case is $2.71 \%$ for the displacement $u_{y}$ and $1.76 \%$ for $r_{x}$ both in case S3, and $1.67 \%$ for $r_{z}$ in case S2. The frequency results present the same trend. The frequencies in which bending motion is predominant are underestimated by the beam model. The largest relative difference is observed for the fixed-free boundary conditions (i.e., 2.75\% in case S3). On the other hand, the frequencies associated with torsion estimated by BECAS are larger than the free-free solutions but smaller than the fixed-free results.

The results show also that the effects of the material anisotropy on the structural response of the beam are correctly captured by the BECAS based beam finite element model. Namely, the torsional motion stemming from the coupling between bending and torsion induced by the material orientation shows very good agreement both in terms of tip rotation and eigenfrequencies.

Regarding the stresses in Figure 6, the effects of the boundary conditions are clearly visible. In the central part of the beam all three models - BECAS, 3DFEM free-free, and 3DFEM fixed-free - show very good agreement. This is clear form the fringe plots obtained at the mid-section of the beam. At this section the boundary conditions do not affect the stresses and the results from BECAS and 3DFEM are practically indistinguishable. The 3DFEM results are for the free-free boundary conditions although the same results were obtained for the fixed-free boundary conditions. At the clamped end of the beam, BECAS deviates significantly from the fixed-free results and approximates the free-free results better.

Finally, note that the BECAS results in Figure 5 show that the elastic axis is oriented at $17.5^{\circ}$ which coincides with the fiber plane orientation. The shear center position is determined based on a truncated expression which does not include the bend-twist coupling term [17]. The shear center position is therefore not affected by the anisotropy introduced by the fiber orientation.

The validation results obtained based on the DTU10MW RWT blade are presented in the Table V and Figures 8 and 11. The eigenfrequencies obtained using the BECAS based beam finite element model and 3D shell finite element model (3DFEM) are in very good agreement, the largest deviation being $1.54 \%$. Note that these frequencies match well also with those reported in [11] obtained using BECAS for the analysis of the cross section properties but relying on a different beam finite element formulation implemented in HAWC2 [2]. The largest difference between these results and the BECAS based beam model presented in this paper was $3 \%$.

Overall the blade deformation results presented in Figure 8 are in good agreement for both load cases. The results suggest that the BECAS model is generally more compliant than the 3DFEM model. This may partly be due to the mesh generation procedure. The cross section finite element mesh used in BECAS was automatically generated through extrusion of the shell finite element model in the thickness direction as illustrated in Figure 9. This procedure may generate small discrepancies in the material distribution between the BECAS and shell finite element model which then affects the stiffness and mass properties and ultimately can be observed in the structural response of the models.

An exception to this behavior is observed in Figure 8(e) where the torsional motion of the blade resulting from transverse flapwise and edgewise forces in load case BLC1 is larger in the 3DFEM model. However, in this case it is difficult to conclude that this is a result of the overestimation of the torsional stiffness by the beam model. The applied transverse forces are not applied at the shear center and a torsional moment is therefore induced locally. The irregularities in the lengthwise variation of the torsional rotation given by the 3DFEM model suggest that these moments induce significant local deformation. The local effect of the loads is only partly captured by the beam model and is perhaps the reason for the deviation between the two models. These local effects are negligible for the other blade deformation results obtained for the same load case as seen in Figure 8(a-d). For the torsional load case, the torsional moment applied at the tip also leads to a large deviation in the results as seen in $8(\mathrm{f})$. The magnitude of the remaining components is too small and therefore omitted.

The effect of the loads is also visible in the strain results presented in Figure 11. For both load cases there is a very good match between the strains obtained by BECAS and the 3DFEM model. Note that, in accordance with the blade deformation results, the strain results for load case BLC1 also indicate that the BECAS model is generally more compliant than the 3DFEM model for both load cases. The strains from BECAS are offset by approximately $1 \%$ everywhere except in the vicinity of the load application points where the deviation is larger. For the torsional load case BLC2 the deviation is practically negligible although increasing significantly close to the tip where the moment is applied.

Finally, note that the strains obtained by BECAS do not account for tapering, twist and spanwise curvature which is naturally accounted for in the 3DFEM model. However, in the inner part of the blade where these geometrical features 
are more pronounced the deviations are not larger, suggesting that their effect on the strains is of minor importance in the regions where the strains were measured.

The last example concerns the analysis of the strains and stresses in a detailed wind turbine blade cross section. The stresses presented in Figure 14 analyzed along the different paths match very closely for all load cases and stress components, the largest deviation being less than 1\%. In Figure 14(a-c) the jumps in the stresses resulting from the different materials across the thickness are correcltly captured by BECAS. The linear variation of the longitudinal stresses along the length of the shear web is visible in Figure 14(d). As expected the shear stress $\sigma_{12}$ is approximately constant along the same path as seen in Figure 14(e). The stresses in Figure 14(g-i) are analyzed along the outermost layer of the airfoil which is composed of a single material - glass fiber triaxial laminate. Note that the non-zero $\sigma_{22}$ stresses in Figure 14(h) are a result of layering materials with different Poisson ratios. The discontinuities of the $\sigma_{22}$ stresses at the outer surface are a result of the material discontinuity in the layers below.

The last results concern the stresses at a the connection between the shear web, cap, and leading edge panel as presented in Figure 15. The analysis of the stresses in this region is specially challenging since it is composed of many different materials (e.g., uniaxial and triaxial laminates, core material, and adhesive) and joins different types of panels (e.g., the monolythic laminates in the caps with the sandwich panels of the leading edge and shear web). The BECAS and the 3DFEM model results show a very good match thus attesting the ability of BECAS to correctly account for different material effects and accurately predict complex 3D strain and stress fields.

In general, the set of results presented in this paper suggests that the proposed framework is suitable for the structural analysis of wind turbine blades. Results both at the cross section and blade length scale show a good agreement with other modeling approaches routinely used in wind turbine blade design. Perhaps one of the biggest challenges lying ahead for the further development of the proposed framework concerns the incorporation of geometrical nonlinearities at the cross section level. Existing blade designs are composed of panels (e.g., in the trailing edge region) which are sufficiently flexible such that deformation stemming from geometrical nonlinearities becomes significant even at low load levels. Future work will focus on the development of a reduced order model which can take this type of nonlinearities into account. The aim is to devise an efficient procedure which can be used, among other, to predict the effect of these nonlinearities on the strains and stresses and estimate panel buckling. However, note that the problem is not trivial and its solution may jeopardize the computational efficiency of the reduced order model.

Finally, the generation of a reduced order model requires a certain amount of computation time. However, once assembled the reduced order model is computationally very efficient and can accurately describe certain properties of a complex system. In the case of the BECAS based beam model presented here, the solution to the equilibrium equations at each cross section are the main source of computational expense. As shown in this paper, the resulting beam finite element model can be used to accurately analyze the local and global response of wind turbine blades. However, the true potential of the model regarding its efficiency is demonstrated only when applied in computationally intensive applications like the time series analysis of the nonlinear aeroelastic response of a wind turbine blade.

\section{CONCLUSIONS}

This paper describes and assesses the accuracy of the BEam Cross section Analysis Software - BECAS - a computational framework for structural analysis of wind turbine blades. The framework is built upon a 2D finite element based cross section analysis tool capable of predicting the effects of material anisotropy and inhomogeneity for sections of arbitrary geometry. A brief presentation of the most important theoretical aspects underlying the cross section and beam finite element analysis procedure was given. Three validation examples were considered - solid square cross section beam, an entire wind turbine blade, and detailed wind turbine blade cross section. The validation work focused on phenomena at the blade length scale (i.e., blade deformation and eigenfrequencies) and cross section length scale (i.e., material strains and stresses). Results generated by the BECAS based beam model were compared with 3D shell and solid finite element models generally showing a very good agreement. Yet, the ability of the BECAS framework to separate the 2D problem at the cross section scale from the blade length scale allows for much greater computational efficiency than that of 3D shell and solid finite element models. Future work includes, among other, the efficient generation of time series of material strains and stresses for reliability and fatigue analysis. These results will give an insight into a number of mechanisms associated with blade failure and perhaps allow for the development of novel technologies for designing blades with improved structural performance.

Finally, it is worth noting that the source code of the entire framework is distributed free of charge for academic use in the hope that it is useful and can be extended at other research institutions. 


\section{ACKNOWLEDGEMENTS}

This first author was funded by the Danish Agency for Science, Technology and Innovation through the Individual Postdoc Grant number 0602-02535B from Det Frie Forskningsråd - Technology and Production, and the Energy Technology Development and Demonstration Programme (EUDP) under contract 64013-0115 Stiffening of Wind Turbine Blades Mitigating leading edge damages. The support is gratefully acknowledged. The second author was partly funded by the Danish Energy Agency through the project EUDP2010-I Light Rotor. The support is gratefully acknowledged. The last author acknowledges the support from the Villum Foundation through the NextTop project.

\section{REFERENCES}

1. Hansen M, Sørensen J, Voutsinas S, Sørensen N, Madsen H. State of the art in wind turbine aerodynamics and aeroelasticity. Progress in Aerospace Sciences Jun 2006; 42(4):285-330.

2. Larsen T, Hansen A. How 2 HAWC2, the user's manual. Denmark. Forskningscenter Ris $\emptyset$. Ris $\emptyset$-R, Ris $\emptyset$ National Laboratory, 2007.

3. Lantz E, Wiser R, Hand M. WP2 - IEA Wind Task 26 - The Past and Future Cost of Wind Energy. Technical Report, IEA Wind 2012.

4. Giavotto V, Borri M, Mantegazza P, Ghiringhelli G, Carmaschi V, Maffioli G, Mussi F. Anisotropic beam theory and applications. Comput Struct 1983; 16(1-4):403-413, doi:10.1016/0045-7949(83)90179-7.

5. Yu W. On Timoshenko-like modeling of initially curved and twisted composite beams. International Journal of Solids and Structures 2002; 39(19):5101-5121.

6. Yu W, Liao L, Hodges D, Volovoi V. Theory of initially twisted, composite, thin-walled beams. Thin-walled structures 2005; 43(8):1296-1311.

7. Chen H, Yu W, Capellaro M. A critical assessment of computer tools for calculating composite wind turbine blade properties. Wind Energy 2010; 13(6):497-516.

8. Blasques JP, Stolpe M. Multi-material topology optimization of laminated composite beam cross sections. Compos Struct 2012; 94(11):3278-3289.

9. Blasques JP. Multi-material topology optimization of laminated composite beams with eigenfrequency constraints. Compos Struct 2014; 111:45-55.

10. Blasques JP, Bitsche RD. An efficient and accurate method for computation of energy release rates in beam structures with longitudinal cracks. Eng Fract Mech 2014; To be accepted.

11. Bak C, Zahle F, Bitsche R, Kim T, Yde A, Henriksen LC, Natarajan A, Hansen M. Description of the DTU 10 MW reference wind turbine. Wind Energy 2014; To be accepted.

12. The DTU 10MW reference wind turbine data repository. http://dtu-10mw-rwt.vindenergi.dtu.dk.

13. Volovoi V, Hodges D, Cesnik C, Popescu B. Assessment of beam modeling methods for rotor blade applications. Mathematical and Computer Modelling 2001; 33(10-11):1099-1112.

14. Jung S, Nagaraj V, Chopra I, Others. Assessment of composite rotor blade modeling techniques. Journal of the American Helicopter Society 1999; 44(3):188.

15. Volovoi VV, Hodges DH, Vam B. Validation of the variational asymptotic beam sectional analysis (VABS). AIAA Journal 2002; 40(10).

16. Bathe KJ. Finite Element Procedures. Prentice Hall: Englewood Cliffs - New Jersey, 1996.

17. Blasques J. User's Manual for BECAS: A cross section analysis tool for anisotropic and inhomogeneous beam sections of arbitrary geometry. Ris $\emptyset$-R, DTU Wind Energy, Denmark, 2012.

18. Dassault Systèmes. Abaqus Analysis User's Manual 2013.

19. Peters ST. Handbook of Composites. Chapman Hall: London, 1998. 


\section{TABLES}

Table I. Catalogue of numerical examples used for validation and corresponding number of degrees of freedom (DOF) in beam (BECAS) and 3D finite element models (3DFEM).

\begin{tabular}{cccc}
\hline & Ref. & DOF BECAS & DOF 3DFEM \\
\hline Square & S1, S2, and S3 & 222 (beam) +3843 (cross section) & 525615 \\
DTU 10 MW RWT blade & DTU10MW & 471 (beam) + & 608970 \\
Detailed WT blade section & DWT & 7869 (average per cross section, 51 cross sections) & 4050369 \\
\hline
\end{tabular}

Table II. Material stiffness and mass properties used for modelling the square and detailed WT blade cross sections. $E_{i j}, G_{i j}$, and $\nu_{i j}$ are elastic modulus, shear modulus, and Poisson coefficient in direction $\{i j\}$, respectively, and $\varrho$ is the mass density. Material properties for square beam are from [19].

\begin{tabular}{|c|c|c|c|c|c|c|}
\hline \multirow[b]{3}{*}{$E_{11}[P a]$} & \multirow{2}{*}{$\frac{\text { Square }}{\text { UD }}$} & \multicolumn{5}{|c|}{ Detailed WT blade section } \\
\hline & & UD & BIAX & TRIAX & Core & Adhesive \\
\hline & $1.43 \times 10^{11}$ & $4.00 \times 10^{10}$ & $1.20 \times 10^{10}$ & $2.00 \times 10^{10}$ & $5.00 \times 10^{7}$ & $3.50 \times 10^{9}$ \\
\hline$E_{22}[P a]$ & $1.00 \times 10^{10}$ & $1.00 \times 10^{10}$ & $1.20 \times 10^{10}$ & $1.00 \times 10^{10}$ & $5.00 \times 10^{7}$ & $3.50 \times 10^{9}$ \\
\hline$E_{33}[\mathrm{~Pa}]$ & $1.00 \times 10^{10}$ & $1.00 \times 10^{10}$ & $1.00 \times 10^{10}$ & $1.00 \times 10^{10}$ & $5.00 \times 10^{7}$ & $3.50 \times 10^{9}$ \\
\hline$G_{12}[P a]$ & $6.00 \times 10^{9}$ & $4.00 \times 10^{9}$ & $1.00 \times 10^{10}$ & $7.50 \times 10^{9}$ & $1.79 \times 10^{7}$ & $1.25 \times 10^{9}$ \\
\hline$G_{13}[P a]$ & $5.00 \times 10^{9}$ & $4.00 \times 10^{9}$ & $3.80 \times 10^{9}$ & $4.00 \times 10^{9}$ & $1.79 \times 10^{7}$ & $1.25 \times 10^{9}$ \\
\hline$G_{23}[P a]$ & $3.00 \times 10^{9}$ & $3.57 \times 10^{9}$ & $3.80 \times 10^{9}$ & $4.00 \times 10^{9}$ & $1.79 \times 10^{7}$ & $1.25 \times 10^{9}$ \\
\hline$\nu_{12}$ & 0.20 & 0.28 & 0.50 & 0.50 & 0.40 & 0.40 \\
\hline$\nu_{13}$ & 0.30 & 0.28 & 0.28 & 0.28 & 0.40 & 0.40 \\
\hline$\nu_{23}$ & 0.52 & 0.40 & 0.28 & 0.28 & 0.40 & 0.40 \\
\hline$\varrho\left[\mathrm{kg} / \mathrm{m}^{3}\right]$ & 2900 & 1900 & 1890 & 1860 & 80 & 1890 \\
\hline
\end{tabular}

Table III. Load cases considered for each of the validation examples presented in Table I. Load case SLC1 is a vertical force applied at the tip of the square beams S1, S2, and S3. Load cases BLC1 and BLC2 are associated with the DTU10MW, where the load application point is the half-chord point of the respective cross section. The torsional moment $m_{z}$ in BLC1 is applied only in the beam finite element model in order to compensate for the offset between the beam nodal positions and the half-chord position. Load case DLC1 and DLC2 associated with the DWT are internal cross section forces and moments defined according to the cross section coordinate system in Figure 12.

\begin{tabular}{|c|c|c|c|c|c|c|c|c|c|c|c|c|}
\hline $\begin{array}{c}\text { Square section } \\
(\mathrm{S} 1, \mathrm{~S} 2, \mathrm{~S} 3)\end{array}$ & SLC1 & $\begin{array}{c}z \\
f_{y}\end{array}$ & $\begin{array}{c}{[\mathrm{m}]} \\
{[\mathrm{kN}]}\end{array}$ & $\begin{array}{c}2 \\
100 \\
\end{array}$ & & & & & & & & \\
\hline \multirow{5}{*}{$\begin{array}{c}\text { DTU 10MW RWT } \\
\text { blade } \\
\text { (DTU10MW) }\end{array}$} & \multirow{4}{*}{ BLC1 } & $z$ & {$[\mathrm{~m}]$} & 20.1 & 30.4 & 33.0 & 47.7 & 52.0 & 62.4 & 65.8 & 76.2 & 84.8 \\
\hline & & $f_{x}$ & {$[\mathrm{kN}]$} & 0.0 & 0.0 & 290.0 & 180.0 & 0.0 & 130.0 & 0.0 & 18.0 & 25.0 \\
\hline & & $f_{y}$ & {$[\mathrm{kN}]$} & 230.0 & 270.0 & 0.0 & 0.0 & 250.0 & 0.0 & 220.0 & 190.0 & 165.0 \\
\hline & & $\left(m_{z}\right)$ & {$[\mathrm{kNm}]$} & -122.7 & -226.5 & -27.1 & -8.0 & -170.8 & -0.6 & -111.9 & -74.0 & -46.9 \\
\hline & BLC2 & $\begin{array}{c}z \\
m_{z} \\
\end{array}$ & $\begin{array}{c}{[\mathrm{m}]} \\
{[\mathrm{kNm}]}\end{array}$ & $\begin{array}{c}89.166 \\
450\end{array}$ & & & & & & & & \\
\hline \multirow{2}{*}{$\begin{array}{l}\text { Detailed WT blade } \\
\text { section (DWT) }\end{array}$} & DLC1 & $\begin{array}{l}T_{y} \\
M_{x}\end{array}$ & {$[\mathrm{MN}]$} & $\begin{array}{c}1 \\
-30\end{array}$ & & & & & & & & \\
\hline & DLC2 & $M_{z}$ & {$[\mathrm{MNm}]$} & 1 & & & & & & & & \\
\hline
\end{tabular}


Table IV. Tip displacement $u_{y}$ and rotations $r_{x}$ and $r_{z}$, and five lowest eigenfrequencies for $2 \mathrm{~m}$ long square beams loaded with tip load $f_{y}=10^{5} \mathrm{~N}$. Results obtained using BECAS based beam model and a 3D solid finite element model in ABAQUS (3DFEM). The fiber angle $\alpha_{f}$ and fiber plane angle $\alpha_{p}$ are defined in Figure 4. Frequencies indicated with $\left({ }^{*}\right)$ correspond to eigenmodes in which torsional motion is predominant. Displacement units are $m$, rotations are in rad, and frequencies are given in $\mathrm{Hz}$. Labels free - free and fixed - free refer to the type of boundary conditions in the 3DFEM model. Relative difference is defined as Rel. Diff. $=\left(v_{3 D F E M}-v_{B E C A S}\right) /\left|v_{3 D F E M}\right| \times 100$ where $v_{3 D F E M}$ and $v_{B E C A S}$ are deformation or frequency values determined using the 3DFEM and BECAS model, respectively.

\begin{tabular}{|c|c|c|c|c|c|c|c|c|c|c|c|}
\hline \multirow{2}{*}{ Ref. } & \multicolumn{2}{|c|}{ Fiber orientation } & & \multicolumn{3}{|c|}{ Tip deformation [m, rad] } & \multicolumn{5}{|c|}{ Frequencies [Hz] } \\
\hline & $\alpha_{p}$ & $\alpha_{f}$ & & $u_{y}$ & $r_{x}$ & $r_{z}$ & $f_{1}$ & $f_{2}$ & $f_{3}$ & $f_{4}$ & $f_{5}$ \\
\hline \multirow{5}{*}{ S1 } & \multirow{5}{*}{$0^{\circ}$} & \multirow{5}{*}{$0^{\circ}$} & BECAS & 0.23 & -0.17 & 0.00 & 27.89 & 27.96 & $157.47^{*}$ & 159.65 & 162.21 \\
\hline & & & 3D FEM (fixed-free) & 0.23 & -0.17 & 0.00 & 27.89 & 27.97 & $157.97 *$ & 159.88 & 162.40 \\
\hline & & & 3D FEM (free-free) & 0.23 & -0.17 & 0.00 & 27.89 & 27.96 & $157.29^{*}$ & 159.67 & 162.22 \\
\hline & & & Rel. Diff. (fixed-free) (\%) & -0.04 & 0.02 & - & 0.03 & 0.03 & 0.32 & 0.14 & 0.11 \\
\hline & & & Rel. Diff. (free-free) (\%) & 0.01 & 0.02 & - & 0.00 & 0.00 & -0.11 & 0.01 & 0.00 \\
\hline \multirow{5}{*}{ S2 } & \multirow{5}{*}{$17.5^{\circ}$} & \multirow{5}{*}{$0^{\circ}$} & BECAS & 0.65 & -0.48 & -0.48 & 16.60 & 16.66 & 99.29 & 101.53 & $181.70^{*}$ \\
\hline & & & 3D FEM (fixed-free) & 0.63 & -0.47 & -0.49 & 16.89 & 17.02 & 101.27 & 104.17 & $183.46^{*}$ \\
\hline & & & 3D FEM (free-free) & 0.65 & -0.48 & -0.48 & 16.62 & 16.68 & 99.93 & 102.53 & $181.33 *$ \\
\hline & & & Rel. Diff. (fixed-free) (\%) & -2.63 & 1.72 & -1.67 & 1.69 & 2.12 & 1.96 & 2.53 & 0.96 \\
\hline & & & Rel. Diff. (free-free) (\%) & -0.14 & 0.01 & 0.00 & 0.11 & 0.15 & 0.64 & 0.98 & -0.20 \\
\hline \multirow{5}{*}{ S3 } & \multirow{5}{*}{$17.5^{\circ}$} & \multirow{5}{*}{$17.5^{\circ}$} & BECAS & 0.65 & -0.48 & -0.46 & 16.60 & 16.65 & 99.43 & 101.43 & $182.15^{*}$ \\
\hline & & & 3D FEM (fixed-free) & 0.63 & -0.47 & -0.47 & 16.85 & 17.07 & 101.24 & 104.30 & $183.64^{*}$ \\
\hline & & & 3D FEM (free-free) & 0.65 & -0.48 & -0.46 & 16.62 & 16.68 & 100.08 & 102.45 & $181.72 *$ \\
\hline & & & Rel. Diff. (fixed-free) (\%) & -2.71 & 1.76 & -1.51 & 1.43 & 2.41 & 1.79 & 2.75 & 0.81 \\
\hline & & & Rel. Diff. (free-free) (\%) & -0.14 & 0.01 & 0.00 & 0.11 & 0.15 & 0.65 & 0.99 & -0.24 \\
\hline
\end{tabular}

Table V. Six lowest eigenfrequencies for DTU 10 MW RWT rotor blade calculated using BECAS and ABAQUS shell finite element model (3D FEM). The labels flapwise (flap), edgewise (edge) and torsional (torsion) are indicative of the predominant motion observed for each of the eigenmodes. Relative difference is defined as Rel. Diff. $=\left(f_{3 D F E M}-f_{B E C A S}\right) /\left|f_{3 D F E M}\right| \times 100$ where $f_{3 D F E M}$ and $f_{B E C A S}$ are the frequencies determined using the 3DFEM and BECAS model, respectively.

\begin{tabular}{ccccccc}
\hline Freqs. [Hz] & $\begin{array}{c}f_{1} \\
\text { (flap) }\end{array}$ & $\begin{array}{c}f_{2} \\
\text { (edge) }\end{array}$ & $\begin{array}{c}f_{3} \\
\text { (flap) }\end{array}$ & $\begin{array}{c}f_{4} \\
\text { (edge) }\end{array}$ & $\begin{array}{c}f_{5} \\
\text { (flap) }\end{array}$ & $\begin{array}{c}f_{6} \\
\text { (torsion) }\end{array}$ \\
\hline BECAS & 0.62 & 0.95 & 1.75 & 2.80 & 3.57 & 5.69 \\
3DFEM & 0.61 & 0.95 & 1.75 & 2.84 & 3.58 & 5.69 \\
Rel. Diff. [\%] & -0.72 & 0.61 & 0.25 & 1.54 & 0.37 & -0.04 \\
\hline
\end{tabular}




\section{FIGURES}

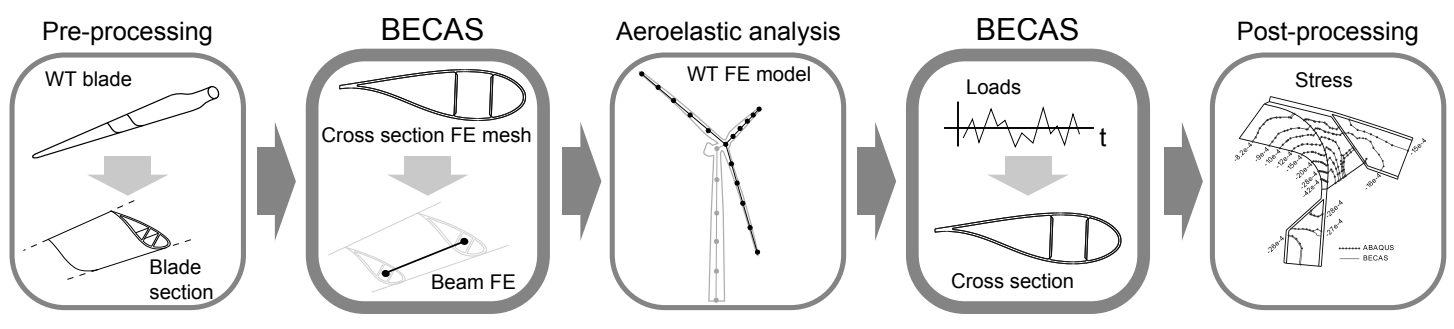

Figure 1. Schematic description of the workflow used for the structural design and analysis of wind turbine blades based on the cross section analysis tool, BECAS.

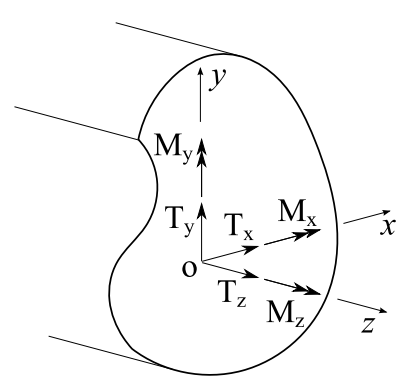

(a) Forces and moments

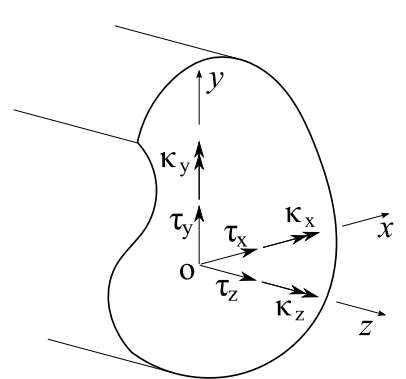

(b) Strains and curvatures

Figure 2. Cross section coordinate system, forces and moments (a) and corresponding strains and curvatures (b) (from [8])

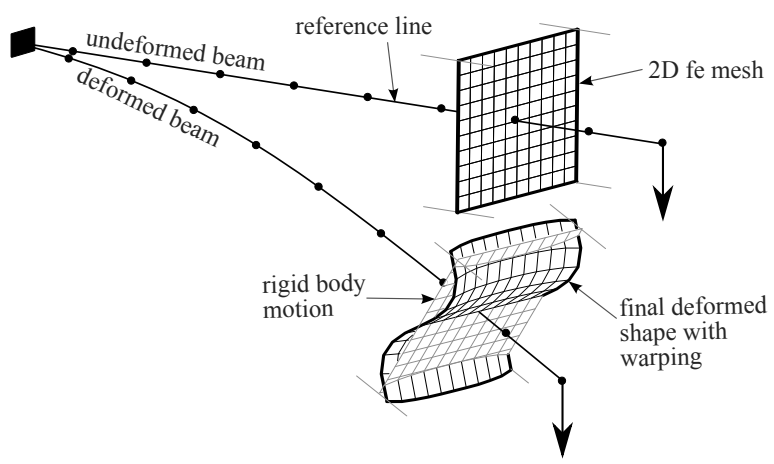

Figure 3. Schematic description of the deformation of a cantilever beam subjected to a tip load. The reference line is meshed using beam finite elements. The deformation of each cross section is described in terms of the rigid body motions and warping displacements. Cross section deformation is analysed using the cross section analysis tool BECAS where a finite element discretization of the cross section geometry is used to approximate the warping deformation (from Blasques and Bitsche [10]). 


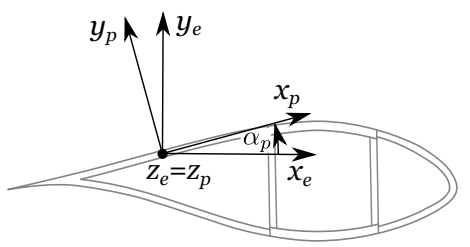

fiber plane angle $\alpha_{p}$

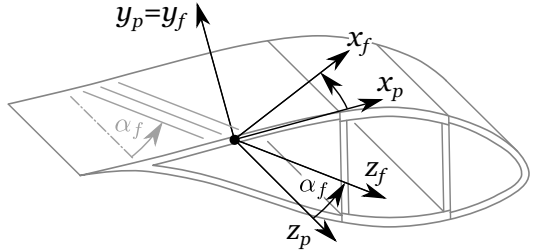

fiber angle $\alpha_{f}$

Figure 4. Definition of fiber plane angle $\alpha_{p}$ and fiber angle $\alpha_{f}$. These angles are used to define the 3D material orientation at each element of the cross section finite element mesh in BECAS.

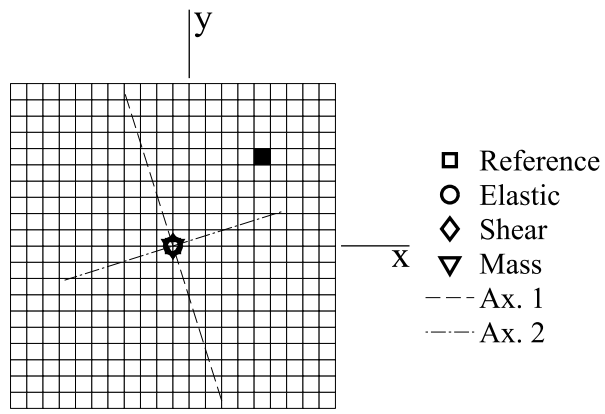

Figure 5. Cross section finite element mesh and location of reference, shear, mass, and elastic centers, and orientation of elastic axes as determined by BECAS for cantilever beam with square cross section S3. Dark element indicates position of the path along which stresses are measured in Figure 6. Stresses are evaluated at element center. 

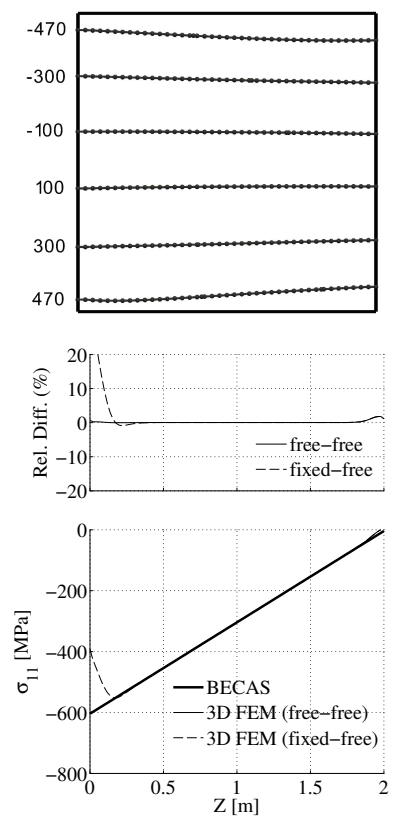

(a) $\sigma_{11}$
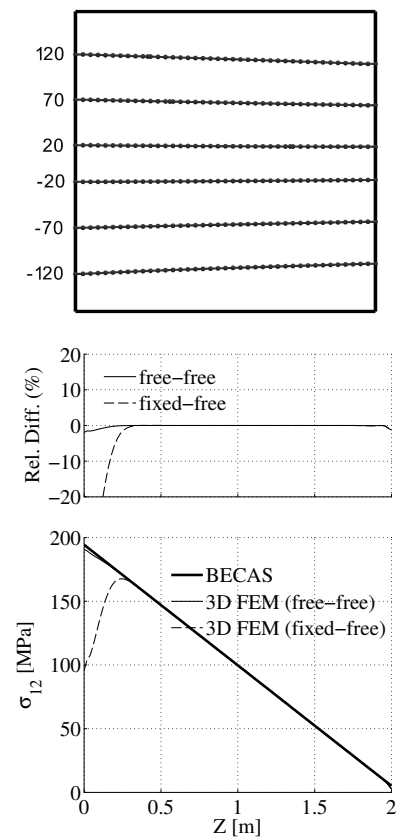

(d) $\sigma_{12}$
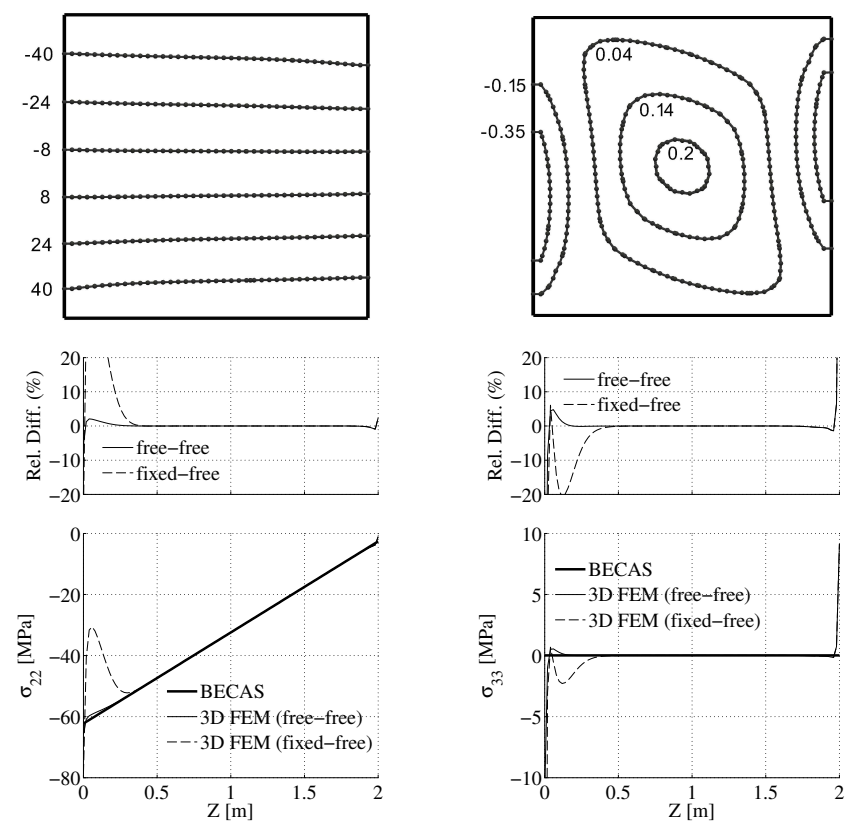

(b) $\sigma_{22}$
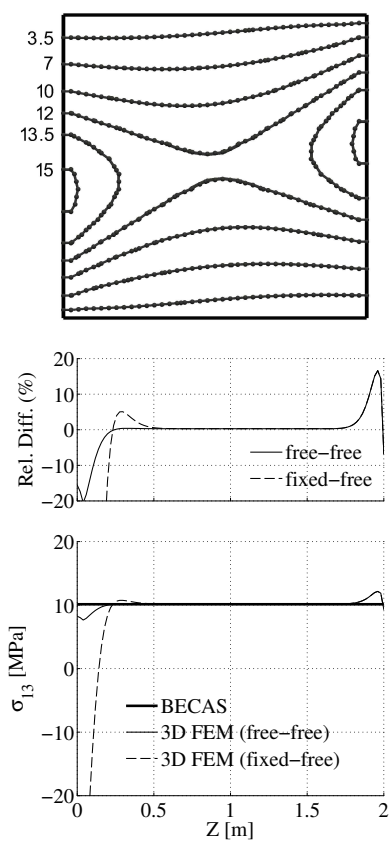

(e) $\sigma_{13}$

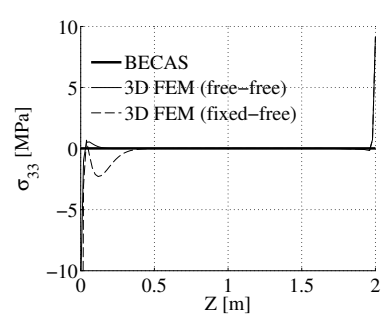

(c) $\sigma_{33}$
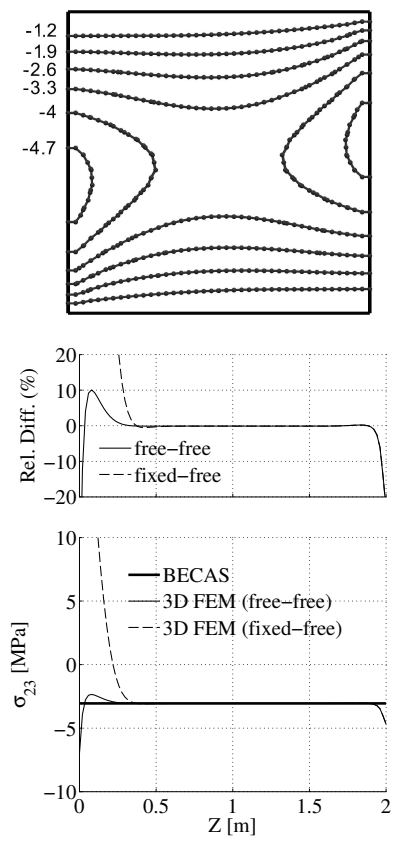

(f) $\sigma_{23}$

Figure 6. Stresses in material coordinate system for cantilever beam S3 obtained using the BECAS based beam finite element model (BECAS) and a 3D solid finite element model (3DFEM). Beam is $2 \mathrm{~m}$ long and subjected to a tip load $f_{y}=10^{5} \mathrm{~N}$. The material orientation is $\alpha_{a}=17.5^{\circ}, \alpha_{p}=17.5^{\circ}$ (cf. Figure 4). Fringe plots refer to element stresses from BECAS (solid line) and 3DFEM with free - free boundary conditions (dotted line) at the cross section at the center of the beam (i.e. $Z=1 \mathrm{~m}$ ). Note that lines from BECAS and 3DFEM overlap. Line plots refer to stresses at a path along the beam length whose position in the cross section is presented in Figure 5. Results for unconstrained and constrainted warping boundary conditions are indicated as free - free and fixed - free, respectively. Relative difference is defined as Rel. Diff. $=\left(\sigma_{3 D F E M}-\sigma_{B E C A S}\right) / \max \left(\left|\sigma_{3 D F E M}\right|\right) \times 100$ where $\sigma_{3 D F E M}$ and $\sigma_{B E C A S}$ are the stresses determined using the 3DFEM and BECAS model, respectively. 


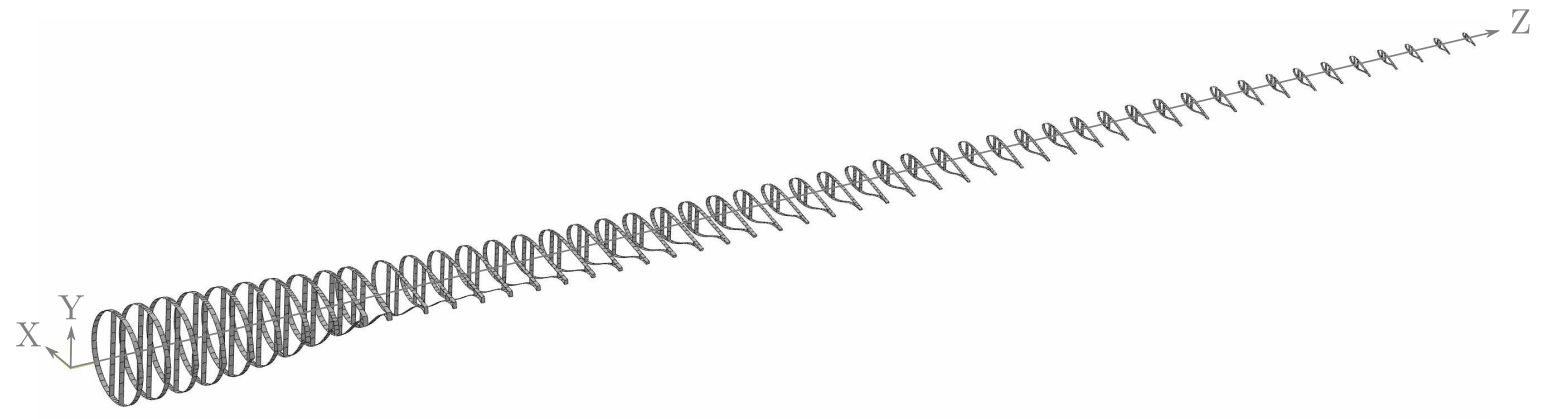

Figure 7. Baseline cross sections and coordinate system of DTU $10 \mathrm{MW}$ reference wind turbine rotor blade.

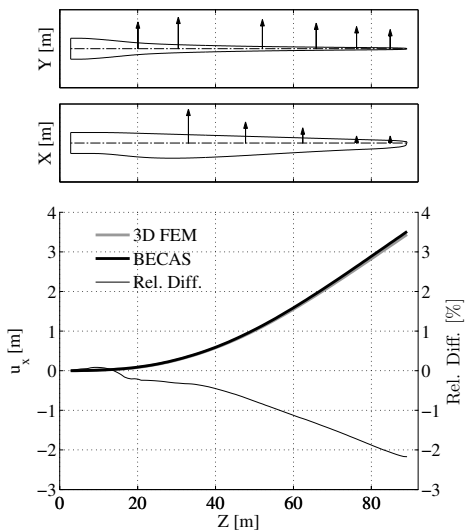

(a) $u_{x}$ for BLC1

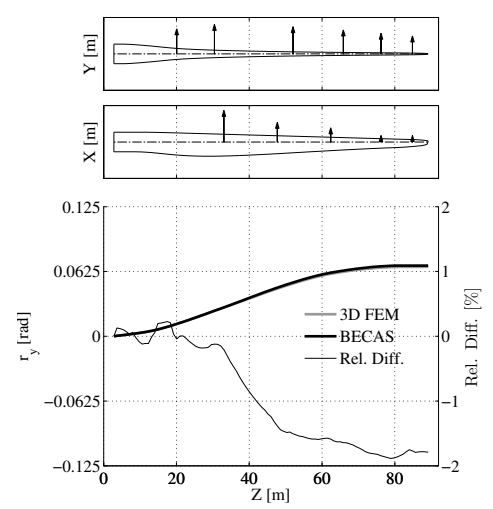

(d) $r_{y}$ for BLC1

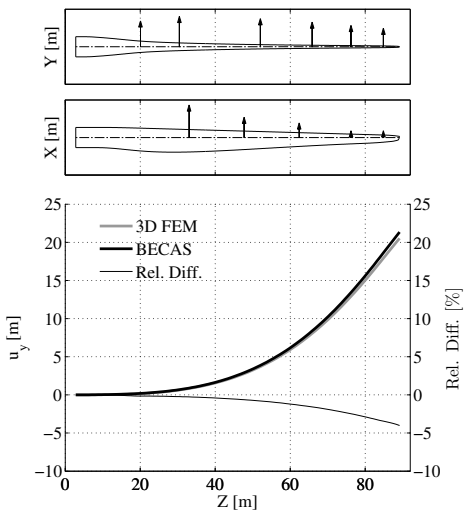

(b) $u_{y}$ for BLC1

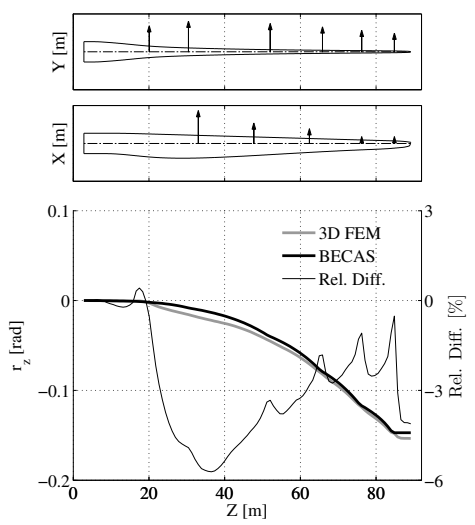

(e) $r_{z}$ for BLC1

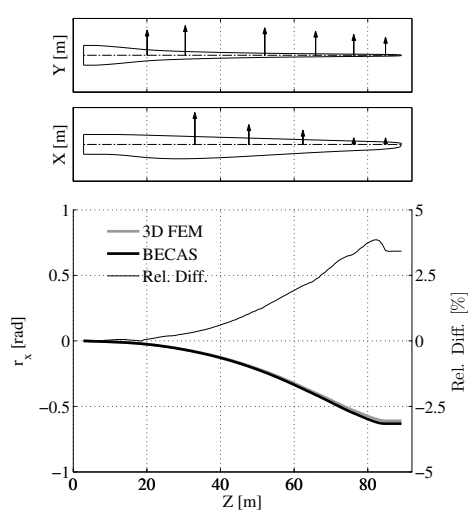

(c) $r_{x}$ for BLC1

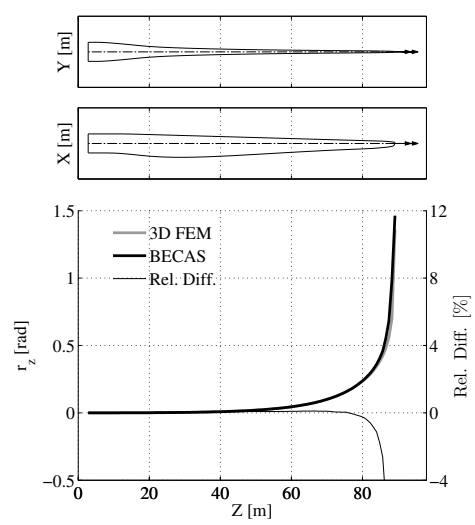

(f) $r_{z}$ for BLC2

Figure 8. Comparison of displacements in the DTU $10 \mathrm{MW}$ reference wind turbine blade determined using BECAS and a shell finite element model in ABAQUS (3DFEM). Results for load case BLC1 (flap and edgewise bending) and BLC2 (torsion), cf. Table III. Relative difference is defined as Rel. Diff. $=\left(d_{3 D F E M}-d_{B E C A S}\right) / \max \left(\left|d_{3 D F E M}\right|\right) \times 100$ where $d_{3 D F E M}$ and $d_{B E C A S}$ are the displacements determined using the 3DFEM and BECAS model, respectively. 


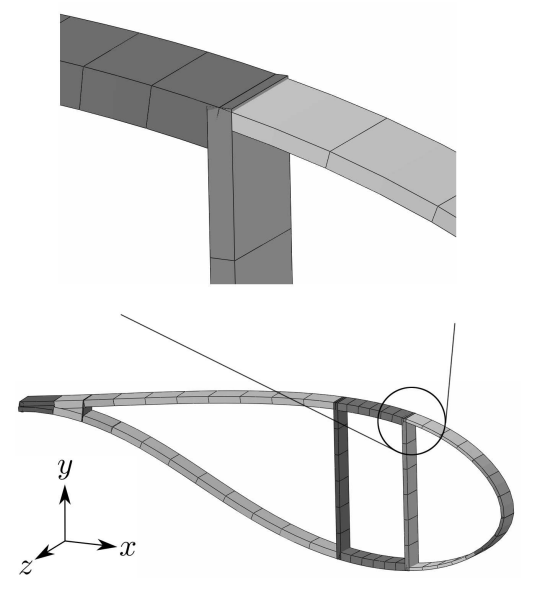

(a) Slice of the finite element shell model (shell thickness visualized).

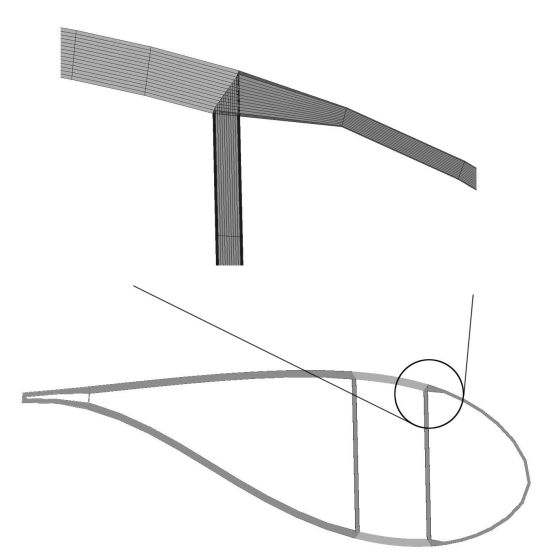

(b) Corresponding BECAS cross section finite element mesh using 14 elements through thickness.

Figure 9. The BECAS cross section finite element mesh (b) is generated automatically based on the shell finite element model (a).

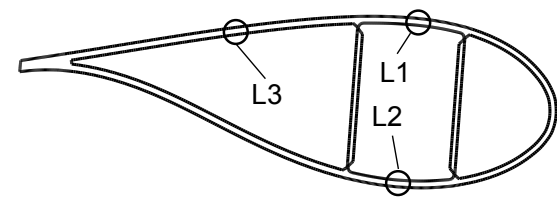

L1 - Cap, suction side, outer layer, along the length;

L2 - Cap, pressure side, outer layer, along the length;

L3 - Trailing edge panel, suction side, outer layer, along the length;

Figure 10. Schematic wind turbine blade section indicating the location of the logintudinal paths used in the analysis of the strains in the DTU10MW RWT rotor blade. Strains along these paths are presented in Figure 11.

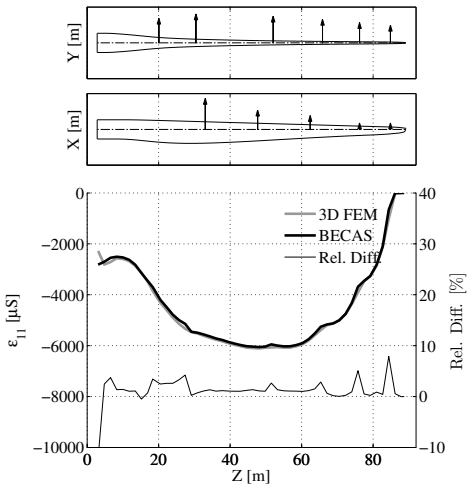

(a) $\epsilon_{11}$ for BLC1 along L1

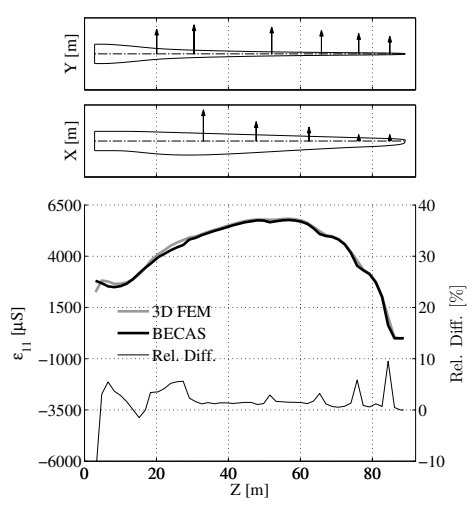

(b) $\epsilon_{11}$ for BLC1 along L2

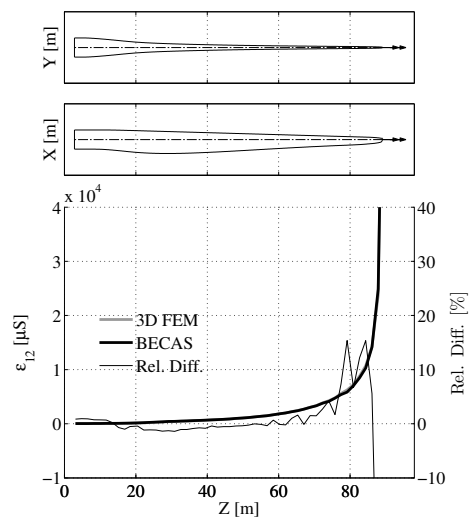

(c) $\epsilon_{12}$ for BLC2 along L3

Figure 11. Strains in the DTU $10 \mathrm{MW}$ reference wind turbine blade determined using BECAS and a shell finite element model in ABAQUS. The location of the longitudinal paths are indicated in Figure 10. Results for load case BLC1 (flap and edgewise bending) and BLC2 (torsion), cf. Table III. (a) and (b) Axial strains $\epsilon_{11}$ for load case BLC1. (b) In-plane shear strains $\epsilon_{12}$ for BLC2. Relative difference is defined as Rel. Diff. $=\left(\epsilon_{3 D F E M}-\epsilon_{B E C A S}\right) / \max \left(\left|\epsilon_{3 D F E M}\right|\right) \times 100$ where $\epsilon_{3 D F E M}$ and $\epsilon_{B E C A S}$ are the strains determined using the 3DFEM and BECAS model, respectively. 


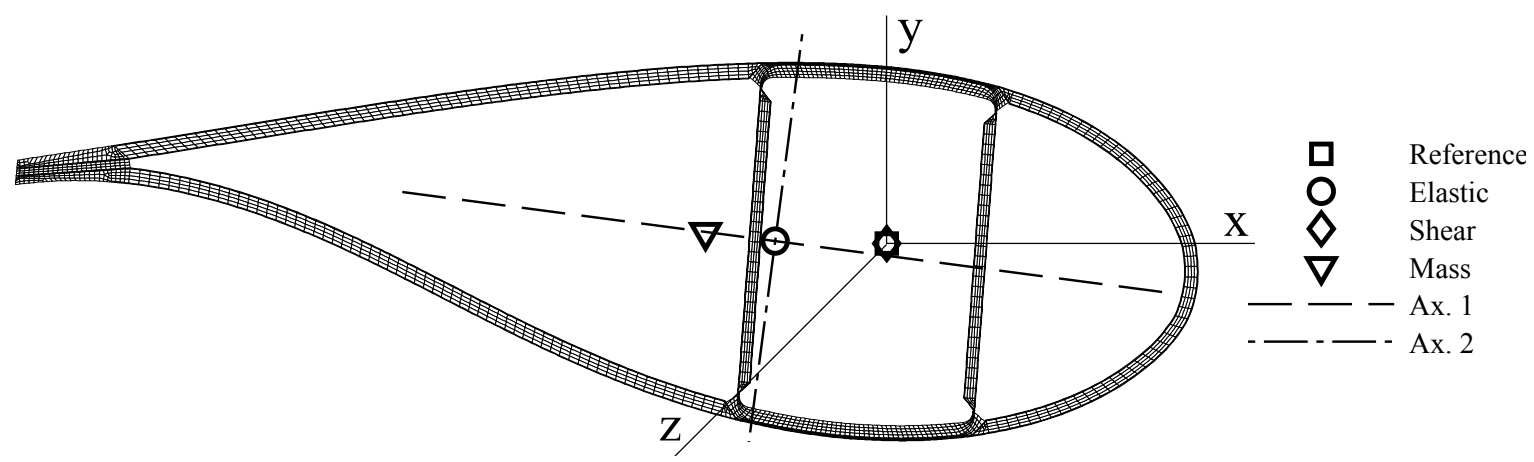

Figure 12. Detailed wind turbine blade cross section (DWT) finite element mesh and coordinate system. Reference, elastic, shear, and mass center positions, and elastic axis orientation as calculated by BECAS.

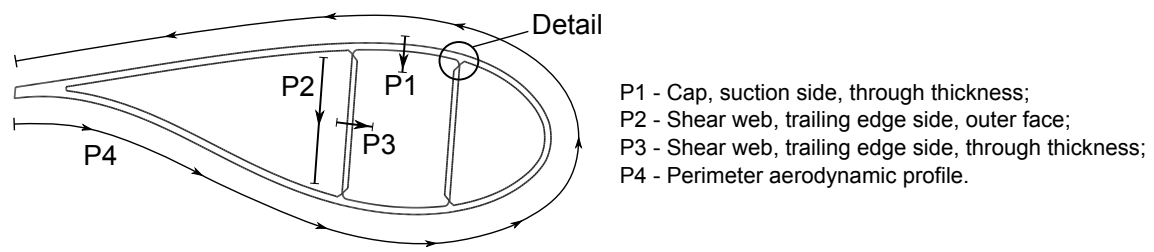

Figure 13. Schematic wind turbine blade section indicating the location of the paths used in the analysis of the stresses in the detailed wind turbine blade cross section (DWT). Stresses along these paths are presented in Figure 14. 


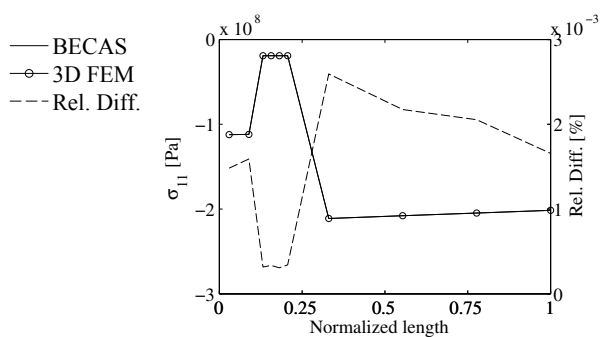

(a) P1 - DLC1

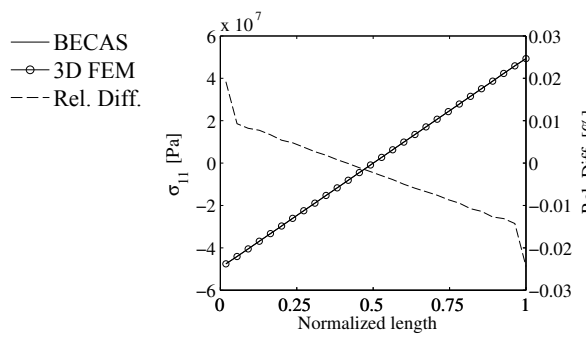

(d) P2 - DLC1

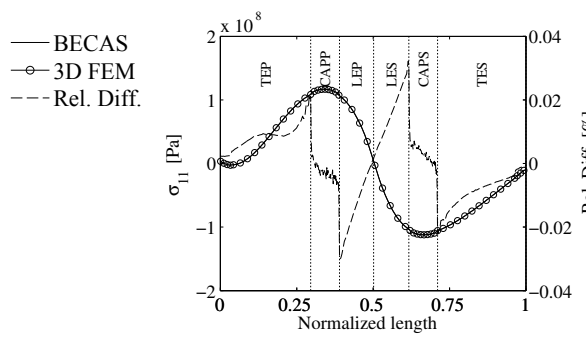

(g) P4 - DLC1

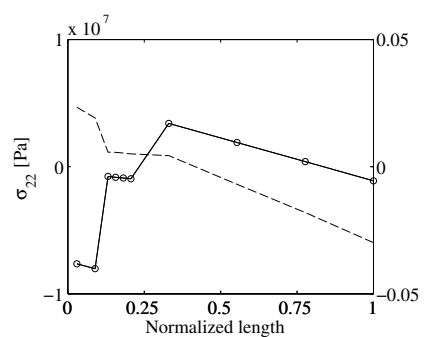

(b) P1 - DLC1

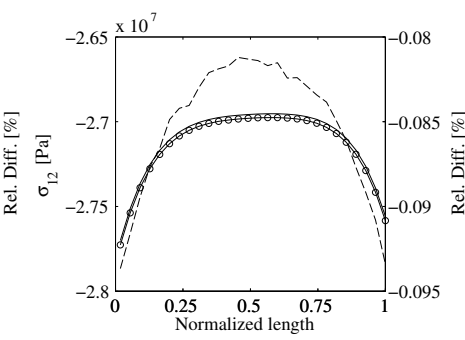

(e) P2 - DLC1

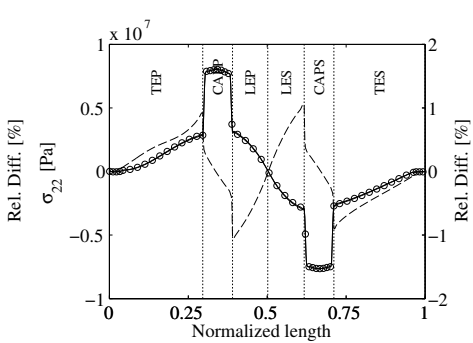

(h) P4 - DLC1

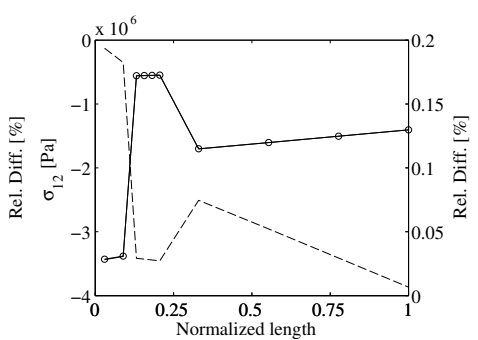

(c) P1 - DLC2

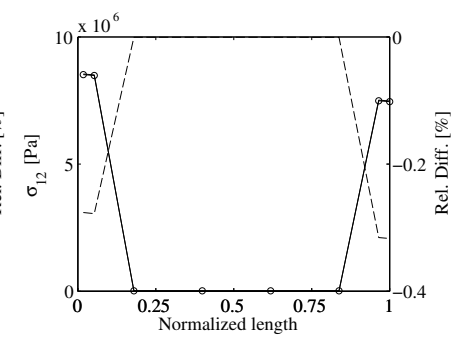

(f) P3 - DLC2

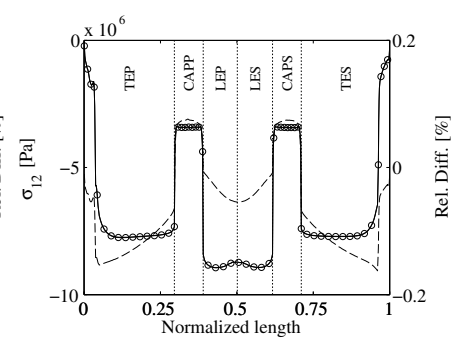

(i) P4 - DLC2

Figure 14. Results by BECAS and 3D FEM for detailed wind turbine blade cross section. Cross section subjected to load cases DLC1 (flapwise bending) and DLC2 (torsion), cf. Table III. Stress components $\sigma_{11}, \sigma_{22}$ and $\sigma_{12}$ evaluated at element centers along paths P1 (spar cap, suction side, through thickness), P2 (shear web, from suction side to pressure side), P3 (shear web, through thickness), P4 (around the perimeter of the aerodynamic profile), cf. Figure 13. For P4 each region is identified: TEP (trailing edge panel pressure side), CAPP (spar cap pressure side), LEP (leading edge panel pressure side), LES (leading edge panel suction side), CAPS (spar cap suction side), TES (trailing edge panel suction side). Relative difference is defined as Rel. Diff. = $\left(\sigma_{3 D F E M}-\sigma_{B E C A S}\right) / \max \left(\left|\sigma_{3 D F E M}\right|\right) \times 100$ where $\sigma_{3 D F E M}$ and $\sigma_{B E C A S}$ are the stresses determined using the 3DFEM and BECAS model, respectively. 


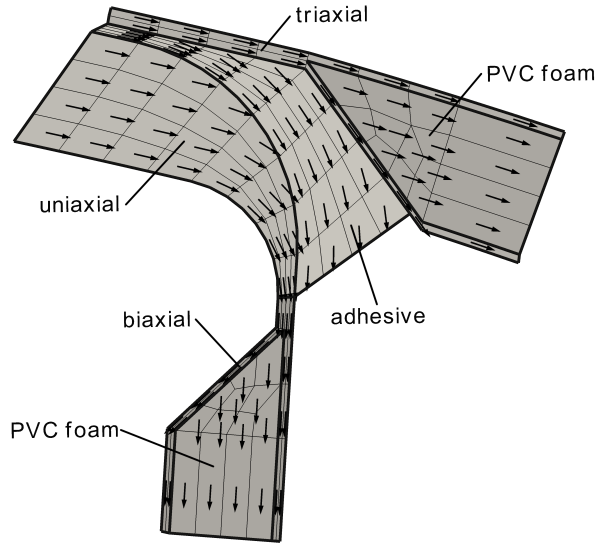

(a)

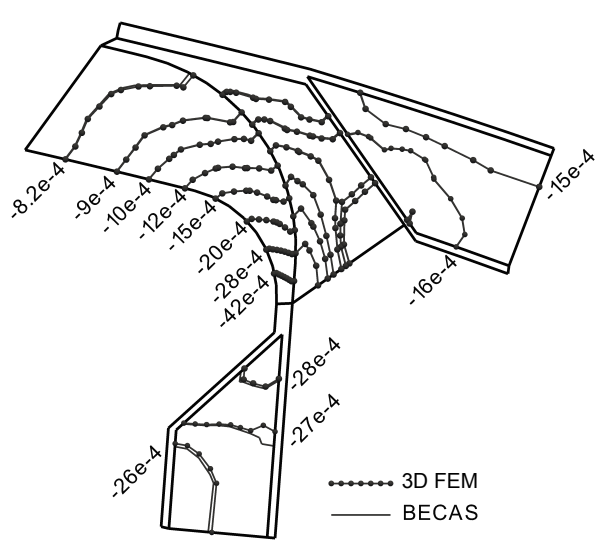

(b)

Figure 15. Results at the junction between the caps, shear webs, and leading edge panels of the detailed cross-section (see Fig. 13). Cross section subjected to load cases DLC1 (flapwise bending), cf. Table III. (a) Material distribution and principal fiber plane orientations. (b) Strains $\epsilon_{12}$ in material coordinate system analyzed at element centroids. 